\title{
General tree-level amplitudes by factorization limits
}

\author{
Kang Zhou ${ }^{\mathrm{a}}$, Chenkai Qiao \\ Zhejiang Institute of Modern Physics, Zhejiang University, Hangzhou 310027, China
}

Received: 7 December 2014 / Accepted: 3 April 2015 / Published online: 24 April 2015

(C) The Author(s) 2015. This article is published with open access at Springerlink.com

\begin{abstract}
To find boundary contributions is a rather difficult problem when applying the BCFW recursion relation. In this paper, we propose an approach to bypass this problem by calculating general tree amplitudes that contain no polynomial using factorization limits. More explicitly, we construct an expression iteratively, which produces the correct factorization limits for all physical poles, and does not contain other poles, then it should be the correct amplitude. To some extent, this approach can be considered as an alternative way to find boundary contributions. To demonstrate our approach, we present several examples: $\phi^{4}$ theory, pure gauge theory, Einstein-Maxwell theory, and Yukawa theory. While the amplitude allows the existence of polynomials which satisfy the correct mass dimension and helicities, this approach is not applicable to determining the full amplitude.
\end{abstract}

\section{Introduction}

The importance of scattering amplitudes can never be overestimated in high-energy physics, for it serves as the intermediary between theories and experiments. The traditional approach for the analytic calculation of scattering amplitudes relies on Feynman diagrams and Feynman rules; it is well systematized and has clear physical pictures. However, with an increasing number of external states, the fast growth in the number of diagrams makes the computation extremely complicated. Naturally, more efficient approaches are desired.

Initiated by Witten's twistor string program [1], many powerful approaches have been developed in the past decade. Among these, the BCFW on-shell recursion relation [2,3] has been successfully applied in many contexts involving massless particles at tree and loop levels, as well as for massive

The unconventional order of authors is merely to satisfy the outdated requirement for Phy. Degree of the school.

a e-mail: zkzrzm@126.com particles at tree level (see for reviews [4-6]). In the derivation of the recursion relation, one deforms a pair of external momenta ${ }^{1}$ in terms of a single complex variable $z$, thus the on-shell amplitude $A(z)$ becomes a rational function of $z$. The behavior of $A(z)$ in the limit $z \rightarrow \infty$ becomes crucial. If $A(z) \rightarrow 0$ when $z \rightarrow \infty$, amplitudes can be reconstructed by summing over residues of poles at finite positions. However, if $A(z)$ does not vanish at infinity, a boundary contribution will emerge. Thus to get the correct amplitudes, we need to find these boundary contributions.

It has been clarified that for many theories, such as gauge theory and gravity, boundary terms can be zero with some proper choices of momentum deformations $[9,10]$. However, there are other theories in which boundary contributions cannot be avoided, for example, $\phi^{4}$ theory and theories with Yukawa couplings [9]. Several attempts have been proposed for finding boundary contributions. The first one is to add auxiliary fields so that boundary terms for the enlarged theory are zero $[11,12]$. By proper reduction one gets the desired amplitudes. The second one is to analyze Feynman diagrams carefully to isolate boundary contributions within these diagrams [13-15]. With this information, boundary terms can be calculated directly or recursively. The third one is to relate boundary terms to the zeros of amplitudes, i.e., roots of amplitudes [16-18]. However, it is not easy to find such zeros. Despite the progress mentioned above, a general effective approach to handle boundary terms is still lacking.

In this paper, we propose an approach to calculate tree amplitudes without polynomials, which avoids the direct computation of boundary contributions. The idea is to seek an expression that is consistent with factorization limits for all physical poles and does not contain other poles. The searching can be done iteratively. We will start with a scalar function which has the correct factorization limits for some poles. This starting function can be obtained by calculating the factorization limit for one channel, or be chosen as the result

$\overline{1}$ There are other deformations; see $[7,8]$. 
given by the $\mathrm{BCFW}$ recursion relation regardless of the existence of boundary contributions. Having this input, at each step we consider the factorization limit for a new channel, and adjust the starting function to include it, without disturbing the correct factorization limits that have been already satisfied. When correct factorization limits for all physical channels are included, we should eliminate possible spurious poles. Then we claim that the correct amplitude is found. This approach disregards boundary contributions, therefore it can be applied to circumstances in which the BCFW approach is difficult. This approach focuses on the pole structures of amplitudes therefore it cannot detect polynomial terms which do not have any pole. Thus, if the amplitude admits polynomials which satisfy correct mass dimension and helicities, this ambiguity will arise and the full amplitudes cannot be determined.

This paper is organized as follows. In Sect. 2 we give a brief overview of this approach. Then we use it to calculate amplitudes of $\phi^{4}$ theory, pure gauge theory, and the EinsteinMaxwell theory, as shown in Sects. 3, 4, and 5, respectively. In these examples, we will not encounter any spurious pole when obtain an expression provides the correct factorization limits for all physical poles. In Sect. 6, we take the four-point amplitude of Yukawa theory as an example to discuss how to remove possible spurious poles. In Sect. 7, a brief conclusion is given.

Throughout this paper, we use the QCD conventions, i.e., $2 k_{i} \cdot k_{j} \equiv\langle i \mid j\rangle[j \mid i]$, and $s_{i j \ldots l}$ denotes $\left(k_{i}+k_{j}+\cdots+k_{l}\right)^{2}$. Also $A_{s_{1} \cdots s_{n}}$ denotes an expression which has the correct factorization limits for the poles $s_{1}, \ldots, s_{n}$. Furthermore, we will neglect the overall factor $i$ in amplitudes, consequently the corresponding factorization is $A \rightarrow-A_{L} \frac{1}{P^{2}} A_{R}$, rather than $A \rightarrow A_{L} \frac{i}{P^{2}} A_{R}$. It also implies we should take

$\left.\operatorname{Res}\left(\frac{A(z)}{z}\right)\right|_{z=z_{\alpha}}=-\sum_{h} A_{L}\left(z_{\alpha}\right) \frac{1}{P^{2}} A_{R}\left(z_{\alpha}\right)$,

when using the BCFW approach.

\section{Outline of the approach}

In this section, we present a brief discussion as regards the approach used in this paper. It bases on the property that a correct amplitude has consistent factorization limits for all physical poles. Since a meromorphic function is uniquely determined by its poles and related residues, if an expression has the correct factorization limits for all physical poles, and it does not contain other poles, the expression is almost the correct amplitude without polynomials that we are seeking. Under this observation, one can reconstruct amplitudes by imposing consistent factorization limits for all physical channels.
To find (or guess) the correct expression, we can start from a scalar function depending on external momenta and helicities, which gives the correct factorization limits for some channels. Such a function can be obtained by direct computation of the factorization limit for one channel. For example, consider the channel $\langle 1 \mid 2\rangle \rightarrow 0$, we can write the initial function as ${ }^{2}$

$A_{\langle 1 \mid 2\rangle}=-\frac{\lim _{\langle 1 \mid 2\rangle \rightarrow 0} A_{L} A_{R}}{s_{12}}=\frac{\lim _{\langle 1 \mid 2\rangle \rightarrow 0} s_{12} A}{s_{12}}$.

Obviously, it has the correct factorization limit for $\langle 1 \mid 2\rangle \rightarrow 0$. The initial function can also be chosen as the result by the BCFW approach regardless of the existence of the boundary contribution. In the former choice, the function provides the correct factorization limit for the corresponding channel. In the latter choice, the function at least provides the correct factorization limits for the poles detected by the BCFW deformation.

At this stage, we need to point out a subtlety of this algorithm. There are many different expressions which are equivalent to each other under some particular factorization limits. For example, under the limit $\langle 1 \mid 2\rangle \rightarrow 0, \frac{\langle 1 \mid 3\rangle}{\langle 1 \mid 4\rangle}=\frac{\langle 2 \mid 3\rangle}{\langle 2 \mid 4\rangle}$, but without imposing the limit, $\frac{\langle 1 \mid 3\rangle}{\langle 1 \mid 4\rangle}$ and $\frac{\langle 2 \mid 3\rangle}{\langle 2 \mid 4\rangle}$ are different. More generally, we will have $f \sim f+\langle 1 \mid 2\rangle g$ for arbitrary functions $f$ and $g$. Thus when we use our algorithm, we need to choose a representative element at each step from the entire equivalent class (category) under some factorization limits.

Having the starting expression, the next step is to consider the factorization limit for a new channel. For instance, we start with (2) and consider another channel, for example $\langle 1 \mid 3\rangle \rightarrow 0$. If

$\lim _{\langle 1 \mid 3\rangle \rightarrow 0} s_{13} A_{\langle 1 \mid 2\rangle}=\lim _{\langle 1 \mid 3\rangle \rightarrow 0} A_{L} A_{R}$,

i.e., $A_{\langle 1 \mid 2\rangle}$ also gives the correct factorization limit for the pole $\langle 1 \mid 3\rangle$, we move on to include the correct factorization limit for another new physical pole. If this fails, we then construct

$A_{\langle 1 \mid 3\rangle}^{\prime}=A_{\langle 1 \mid 2\rangle}+\left(A_{\langle 1 \mid 3\rangle}-\frac{\lim _{\langle 1 \mid 3\rangle \rightarrow 0} s_{13} A_{\langle 1 \mid 2\rangle}}{s_{13}}\right)$,

where

$A_{\langle 1 \mid 3\rangle}=-\frac{\lim _{\langle 1 \mid 3\rangle \rightarrow 0} A_{L} A_{R}}{s_{13}}$.

Now we need to see if $A_{\langle 1 \mid 3\rangle}^{\prime}$ has the correct factorization limit for $\langle 1 \mid 2\rangle \rightarrow 0$. If it does, we are content and move on to a new pole. If it does not, it means the original expressions, $A_{\langle 1 \mid 2\rangle}$ or $A_{\langle 1 \mid 3\rangle}$ or both, are not proper choices. We need to deform them properly, i.e., to adopt different representations as discussed in the previous paragraph. The goal is that while

${ }^{2}$ It is not necessary to sum over helicities of the on-shell internal line, since to get non-zero three-point sub-amplitudes under the limit $\langle 1 \mid 2\rangle \rightarrow 0$, only one kind of helicity configurations is allowed. 
it gives the correct factorization limit for the new pole, it also keeps the correct factorization limits for the poles in the earlier steps. Although we do not have a general guidance for the choice of proper expressions, in the following sections, we will use many examples to demonstrate how to make efficient choices. However, choices in these examples depend on specific theories, it is not yet clear that whether analogous choices can be applied universally to any theory. ${ }^{3}$

Iterating the procedure above, we can include at least one new pole at each step. Since with proper choices of representative expressions, the set of poles that have correct factorization limits is enlarged, within finite steps, we will obtain a result that has correct factorization limits for all physical poles.

It is possible that the obtained expression also contains spurious poles. One approach to eliminate them is use our framework (4) again. Let us assume that in (4), $A_{\langle 1 \mid 2\rangle}$ has the correct factorization limits for all physical poles and contains a spurious pole $\langle 1 \mid 3\rangle$. In such a case, we have $A_{\langle 1 \mid 3\rangle}=0$. To continue, notice that in (4), not only $A_{\langle 1 \mid 2\rangle}$ and $A_{\langle 1 \mid 3\rangle}$ can be deformed, but also the expression of $\lim _{\langle 1 \mid 3\rangle \rightarrow 0} s_{13} A_{\langle 1 \mid 2\rangle}$ is not unique. Thus we can deform $\lim _{\langle 1 \mid 3\rangle \rightarrow 0} s_{13} A_{\langle 1 \mid 2\rangle}$ under the limit $\langle 1 \mid 3\rangle \rightarrow 0$, so that $\frac{\lim _{\langle 1 \mid 3\rangle \rightarrow 0} s_{13} A_{\langle 1 \mid 2\rangle}}{s_{13}}$ does not contain any physical pole. Then we get the result $A_{\langle 1 \mid 2\rangle}^{\prime}$ which has the correct factorization limits for all physical poles, while the spurious pole $\langle 1 \mid 3\rangle$ has been excluded. Again, although we will demonstrate this technique in examples, there is no general guidance of how to deform $\lim _{\langle 1 \mid 3\rangle \rightarrow 0} s_{13} A_{\langle 1 \mid 2\rangle}$ correctly. Iterating this procedure to remove all spurious poles, we find the full amplitude as desired.

It is worth noticing that this approach is based on the assumption that the amplitude does not contain any polynomial. If an amplitude contains a polynomial that has no pole, for instance a constant, this term cannot be detected by any factorization limit. An example is adding a $\phi^{6}$ term in the original $\phi^{4}$ Lagrangian, the $\phi^{6}$ term adds a constant term into the six-point amplitude of pure scalars, then such an amplitude cannot be fully calculated by our approach. However, all examples computed in the following sections do not contain any polynomial term. We will give a brief proof for the absence of polynomial terms in Appendix A.

The calculation of this approach is more complicated than the BCFW one since all possible factorization channels need to be considered, and expressions of factorization limits also need to be fixed. However, since factorization is a general

\footnotetext{
${ }^{3}$ Although taking efficient choices will simplify the calculation, one can try to achieve this goal 'blindly' by using the following observation: The uncertainty is due to the rational function of mass dimension zero, which is helicity neutral for all external particles and reduces to 1 in the factorization limit. Thus one can construct basis of such rational functions and fix their coefficients using other factorization limits. This is only a tentative suggestion, which is beyond the scope of this manuscript and we will leave it to future work.
}

property of amplitudes, this approach can be applied to any quantum field theories.

\section{Example 1: $\phi^{4}$ theory}

Given the general framework in the previous section, let us consider a simplest example, the color-ordered massless $\lambda \phi^{4}$ theory. In this theory, the lowest-point amplitude is given by

$A_{4}(1,2,3,4)=-\lambda$.

From now on we will drop out the coupling constant $-\lambda$. We will show how to construct amplitudes of the theory by our approach. Results in this section will be the same as those given in [13]. Here, the starting expression will be obtained by the BCFW approach. Notice that the missing boundary terms will be detected, although we do not pay attention to them.

\subsection{The six-point amplitude $A_{6}(1,2,3,4,5,6)$}

With only the $\phi^{4}$ interaction, only amplitudes with an even number of external particles can exist. The first nontrivial amplitude is $A_{6}(1,2,3,4,5,6)$. Under the deformation

$\lambda_{1} \rightarrow \lambda_{1}-z \lambda_{2}, \quad \tilde{\lambda}_{2} \rightarrow \tilde{\lambda}_{2}+z \tilde{\lambda}_{1}$,

there is only one pole $s_{561}$ detected and the corresponding residue gives

$A_{0}=-\frac{1}{S_{561}}$,

which is our starting expression for the iterative construction. Obviously, $A_{0}$ has the correct factorization limit for $s_{561} \rightarrow$ 0 .

The physical amplitude also contains poles $s_{123}$ and $s_{612}$, for which $A_{0}$ cannot give the correct factorization limits. Under the limit $s_{123} \rightarrow 0$, we have $\lim _{s_{123} \rightarrow 0} s_{123} A_{6}(1,2,3$, $4,5,6)=-1$, but $\lim _{s_{123} \rightarrow 0} s_{123} A_{0}=0$, thus we need to add $\frac{-1}{s_{123}}$ to $A_{0}$ to get the expression $\left(\frac{-1}{s_{561}}+\frac{-1}{s_{123}}\right)$ at the second step. Now it has the correct factorization limits for the poles $s_{561}$ and $s_{123}$, but not for the pole $s_{612}$. Analogously, we add a new term $\frac{-1}{s_{612}}$ to get

$A_{6}(1,2,3,4,5,6)=-\left(\frac{1}{s_{561}}+\frac{1}{s_{612}}+\frac{1}{s_{123}}\right)$.

Since all factorization limits of possible channels have been given correctly, and no spurious pole appears (we will not emphasize the verification of the existence of spurious poles again if an expression does not contain any spurious pole), (9) is the correct result. Although we did not try to find the boundary term, the added terms in these steps give the boundary contribution $\left(\frac{-1}{s_{612}}+\frac{-1}{s_{123}}\right)$. 
3.2 The eight-point amplitude $A_{8}(1,2,3,4,5,6,7,8)$

The second example is the eight-point amplitude $A_{8}(1,2,3$, $4,5,6,7,8)$. Under the $\langle 1| 2]$-shift, the BCFW approach gives

$A_{0}=\left[\frac{1}{s_{781}}\left(\frac{1}{s_{234}}+\frac{1}{s_{345}}+\frac{1}{s_{456}}\right)+\frac{1}{s_{234}}\left(\frac{1}{s_{567}}+\frac{1}{s_{678}}\right)\right]$,

which gives the correct factorization limits for the poles $s_{781}$ and $s_{234}$ detected by the deformation.

$A_{0}$ does not contain the pole $s_{123}$, which indicates

$$
\frac{\lim _{s_{123} \rightarrow 0} s_{123} A_{0}}{s_{123}}=0 \text {. }
$$

Hence when we consider the factorization limit for the pole $s_{123}$, a new term needs to be added,

$$
\begin{aligned}
A_{s_{123}} & =-\frac{\lim _{s_{123} \rightarrow 0} A_{4}\left(1,2,3,-P_{123}\right) A_{6}\left(P_{123}, 4,5,6,7,8\right)}{s_{123}} \\
& =\frac{1}{s_{123}}\left(\frac{1}{s_{456}}+\frac{1}{s_{567}}+\frac{1}{s_{678}}\right) .
\end{aligned}
$$

Thus we obtain $A_{1}=A_{0}+A_{s_{123}}$ at the second step, which gives correct factorization limits for the poles $s_{123}, s_{781}$, and $s_{234}$.

But $A_{1}$ does not contain the pole $s_{812}$, similarly we need to add

$$
A_{s_{812}}=\frac{1}{s_{812}}\left(\frac{1}{s_{345}}+\frac{1}{s_{456}}+\frac{1}{s_{567}}\right)
$$

to get $A_{2}=A_{1}+A_{s_{812}}$, which has the correct factorization limits for poles $s_{781}, s_{234}, s_{123}$, and $s_{812}$.

Next we consider the factorization limit for the pole $s_{678} \rightarrow 0$, given by

$A_{s_{678}}=\frac{1}{s_{678}}\left(\frac{1}{s_{123}}+\frac{1}{s_{234}}+\frac{1}{s_{345}}\right)$.

On the other hand, we have

$$
\lim _{s_{678} \rightarrow 0} s_{678} A_{2}=\frac{1}{s_{123}}+\frac{1}{s_{234}} \text {. }
$$

Thus using the adjustment (4), we add the difference between (14) and (15) to get

$A_{3}=A_{2}+\left(A_{s_{678}}-\frac{\lim _{s_{678} \rightarrow 0} s_{678} A_{2}}{s_{678}}\right)=A_{2}+\frac{1}{s_{678}} \frac{1}{s_{345}}$.

It can be checked that $A_{3}$ provides the correct factorization limits for all possible channels, for instance,

$$
\begin{aligned}
& \lim _{s_{345} \rightarrow 0} s_{345} A_{3}=\frac{1}{s_{678}}+\frac{1}{s_{781}}+\frac{1}{s_{812}} \\
& =-\lim _{s_{345} \rightarrow 0} A_{L}\left(3,4,5,-P_{345}\right) A_{R}\left(P_{345}, 6,7,8,1,2\right) .
\end{aligned}
$$

Therefore we have found the correct result

$$
\begin{aligned}
A_{8}(1,2,3,4,5,6,7,8)= & A_{3} \\
= & \sum_{\sigma \in Z_{8}}\left(\frac{1}{s_{\sigma(1) \sigma(2) \sigma(3) s_{\sigma(6) \sigma(7) \sigma(8)}}}\right), \\
& \left.+\frac{1}{2 s_{\sigma(1) \sigma(2) \sigma(3)} s_{\sigma(5) \sigma(6) \sigma(7)}}\right)
\end{aligned}
$$

where the boundary term of $A_{0}$ is

$$
\begin{aligned}
B= & A_{s_{123}}+A_{s_{812}}+\frac{1}{s_{678}} \frac{1}{s_{345}}=\frac{1}{s_{123}}\left(\frac{1}{s_{456}}+\frac{1}{s_{567}}+\frac{1}{s_{678}}\right) \\
& +\frac{1}{s_{812}}\left(\frac{1}{s_{345}}+\frac{1}{s_{456}}+\frac{1}{s_{567}}\right)+\frac{1}{s_{678}} \frac{1}{s_{345}} .
\end{aligned}
$$

3.3 The ten-point amplitude $A_{10}(1,2,3,4,5,6,7,8,9,10)$

Now we consider the third example, the ten-point amplitude $A_{10}(1,2,3,4,5,6,7,8,9,10)$. Using the $\left.\langle 1| 2\right]$-shift, we get the starting expression

$$
\begin{aligned}
A_{0}= & \frac{1}{s_{234}}\left[\frac{1}{s_{12345}}\left(\frac{1}{s_{678}}+\frac{1}{s_{789}}+\frac{1}{s_{89(10)}}\right)\right. \\
& +\frac{1}{s_{56789}}\left(\frac{1}{s_{567}}+\frac{1}{s_{678}}+\frac{1}{s_{789}}\right) \\
& \left.+\frac{1}{s_{567}}\left(\frac{1}{s_{89(10)}}+\frac{1}{s_{9(10) 1}}\right)\right] \\
& +\frac{1}{s_{9(10) 1}}\left[\frac{1}{s_{34567}}\left(\frac{1}{s_{345}}+\frac{1}{s_{456}}+\frac{1}{s_{567}}\right)\right. \\
& +\frac{1}{s_{45678}}\left(\frac{1}{s_{456}}+\frac{1}{s_{567}}+\frac{1}{s_{678}}\right) \\
& \left.+\frac{1}{s_{678}}\left(\frac{1}{s_{234}}+\frac{1}{s_{345}}\right)\right]+\frac{1}{s_{23456}}\left(\frac{1}{s_{234}}+\frac{1}{s_{345}}+\frac{1}{s_{456}}\right) \\
& \times\left(\frac{1}{s_{789}}+\frac{1}{s_{89(10)}}+\frac{1}{s_{9(10) 1}}\right),
\end{aligned}
$$

which has the correct factorization limits for the poles $s_{234}$, $s_{9(10) 1}$, and $s_{23456}$ detected by the deformation.

Since $A_{0}$ does not contain the pole $s_{123}$, we should add a term to $A_{0}$ to provide the correct factorization limit. Similar manipulations as previous lead to $A_{1}=A_{0}+A_{s_{123}}$ where

$$
\begin{aligned}
A_{s_{123}}= & \frac{1}{s_{123}}\left[\frac{1}{s_{56789}}\left(\frac{1}{s_{567}}+\frac{1}{s_{678}}+\frac{1}{s_{789}}\right)\right. \\
& +\frac{1}{s_{45678}}\left(\frac{1}{s_{456}}+\frac{1}{s_{567}}+\frac{1}{s_{678}}\right) \\
& +\frac{1}{s_{12345}}\left(\frac{1}{s_{678}}+\frac{1}{s_{789}}+\frac{1}{s_{89(10)}}\right) \\
& \left.+\frac{1}{s_{456}} \frac{1}{s_{789}}+\frac{1}{s_{456}} \frac{1}{s_{89(10)}}+\frac{1}{s_{567}} \frac{1}{s_{89(10)}}\right] .
\end{aligned}
$$


The new $A_{1}$ has the correct factorization limits for the poles $s_{234}, s_{9(10) 1}, s_{23456}$, and $s_{123}$.

Now we move on to consider the pole $s_{(10) 12}$. After a little computation, we get $A_{2}=A_{1}+A_{s_{(10) 12}}$, where

$$
\begin{aligned}
A_{s_{(10) 12}}= & \frac{1}{s_{(10) 12}}\left[\frac{1}{s_{45678}}\left(\frac{1}{s_{456}}+\frac{1}{s_{567}}+\frac{1}{s_{678}}\right)\right. \\
& +\frac{1}{s_{34567}}\left(\frac{1}{s_{345}}+\frac{1}{s_{456}}+\frac{1}{s_{567}}\right) \\
& +\frac{1}{s_{56789}}\left(\frac{1}{s_{567}}+\frac{1}{s_{678}}+\frac{1}{s_{789}}\right) \\
& \left.+\frac{1}{s_{345}} \frac{1}{s_{678}}+\frac{1}{s_{345}} \frac{1}{s_{789}}+\frac{1}{s_{456}} \frac{1}{s_{789}}\right],
\end{aligned}
$$

which provides the correct factorization limits for the poles $s_{234}, s_{9(10) 1}, s_{23456}, s_{123}$, and $s_{(10) 12}$.

Now we consider the pole $s_{345}$. The correct factorization limit is

$$
\begin{aligned}
A_{s_{345}}= & \frac{1}{s_{345}}\left[\frac{1}{s_{23456}}\left(\frac{1}{s_{789}}+\frac{1}{s_{89(10)}}+\frac{1}{s_{9(10) 1}}\right)\right. \\
& +\frac{1}{s_{12345}}\left(\frac{1}{s_{678}}+\frac{1}{s_{789}}+\frac{1}{s_{89(10)}}\right) \\
& +\frac{1}{s_{34567}}\left(\frac{1}{s_{89(10)}}+\frac{1}{s_{9(10) 1}}+\frac{1}{s_{(10) 12}}\right) \\
& \left.+\frac{1}{s_{678}} \frac{1}{s_{9(10) 1}}+\frac{1}{s_{678}} \frac{1}{s_{(10) 12}}+\frac{1}{s_{789}} \frac{1}{s_{(10) 12}}\right],
\end{aligned}
$$

while $A_{2}$ gives

$$
\begin{aligned}
\lim _{s_{345} \rightarrow 0} s_{345} A_{2}= & \frac{1}{s_{23456}}\left(\frac{1}{s_{789}}+\frac{1}{s_{89(10)}}+\frac{1}{s_{9(10) 1}}\right) \\
& +\frac{1}{s_{34567}}\left(\frac{1}{s_{9(10) 1}}+\frac{1}{s_{(10) 12}}\right) \\
& +\frac{1}{s_{678}} \frac{1}{s_{9(10) 1}}+\frac{1}{s_{678}} \frac{1}{s_{(10) 12}}+\frac{1}{s_{789}} \frac{1}{s_{(10) 12}} .
\end{aligned}
$$

Adding the difference, we can construct

$$
\begin{aligned}
A_{3}= & A_{2}+\left(A_{s_{345}}-\frac{\lim _{s_{345} \rightarrow 0} s_{345} A_{2}}{s_{345}}\right) \\
= & A_{2}+\frac{1}{s_{345}}\left[\frac{1}{s_{12345}}\left(\frac{1}{s_{678}}+\frac{1}{s_{789}}+\frac{1}{s_{89(10)}}\right)\right. \\
& \left.+\frac{1}{s_{89(10)}} \frac{1}{s_{34567}}\right] .
\end{aligned}
$$

Then $A_{3}$ provides the correct factorization limits for the poles $s_{234}, s_{9(10) 1}, s_{23456}, s_{123}, s_{(10) 12}$, and $s_{345}$. Finally we consider the pole $s_{89(10)}$, whose correct factorization limit is

$$
\begin{aligned}
A_{s_{89(10)}}= & \frac{1}{s_{89(10)}}\left[\frac{1}{s_{23456}}\left(\frac{1}{s_{234}}+\frac{1}{s_{345}}+\frac{1}{s_{456}}\right)\right. \\
& +\frac{1}{s_{12345}}\left(\frac{1}{s_{123}}+\frac{1}{s_{234}}+\frac{1}{s_{345}}\right) \\
& +\frac{1}{s_{34567}}\left(\frac{1}{s_{345}}+\frac{1}{s_{456}}+\frac{1}{s_{567}}\right) \\
& \left.+\frac{1}{s_{123}} \frac{1}{s_{456}}+\frac{1}{s_{123}} \frac{1}{s_{567}}+\frac{1}{s_{234}} \frac{1}{s_{567}}\right],
\end{aligned}
$$

while $A_{3}$ gives

$$
\begin{aligned}
\lim _{s_{89(10) \rightarrow 0} s_{89(10)} A_{3}=} & \frac{1}{s_{23456}}\left(\frac{1}{s_{234}}+\frac{1}{s_{345}}+\frac{1}{s_{456}}\right) \\
& +\frac{1}{s_{12345}}\left(\frac{1}{s_{123}}+\frac{1}{s_{234}}+\frac{1}{s_{345}}\right) \\
& +\frac{1}{s_{34567}} \frac{1}{s_{345}}+\frac{1}{s_{123}} \frac{1}{s_{456}} \\
& +\frac{1}{s_{123}} \frac{1}{s_{567}}+\frac{1}{s_{234}} \frac{1}{s_{567}},
\end{aligned}
$$

thus we can construct

$$
\begin{aligned}
A_{4} & =A_{3}+\left(A_{s_{89(10)}}-\frac{\lim _{s_{89(10)} \rightarrow 0} s_{89(10)} A_{3}}{s_{89(10)}}\right) \\
& =A_{3}+\frac{1}{s_{89(10)}}\left[\frac{1}{s_{34567}}\left(\frac{1}{s_{456}}+\frac{1}{s_{567}}\right)\right] .
\end{aligned}
$$

One can verify that $A_{4}$ gives the correct factorization limits for all channels. Hence, we have found the final result,

$$
\begin{aligned}
A_{4}= & \sum_{\sigma \in Z_{10}}\left(\frac{1}{s_{\sigma(1) \sigma(2) \sigma(3)} S_{\sigma(1) \sigma(2) \sigma(3) \sigma(4) \sigma(5)} S_{\sigma(8) \sigma(9) \sigma(10)}}\right. \\
& +\frac{1}{S_{\sigma(1) \sigma(2) \sigma(3)} S_{\sigma(1) \sigma(2) \sigma(3) \sigma(4) \sigma(5)} S_{\sigma(7) \sigma(8) \sigma(9)}} \\
& +\frac{1}{S_{\sigma(1) \sigma(2) \sigma(3)} S_{\sigma(10) \sigma(1) \sigma(2) \sigma(3) \sigma(4)} S_{\sigma(7) \sigma(8) \sigma(9)}} \\
& +\frac{1}{2 s_{\sigma(1) \sigma(2) \sigma(3)} S_{\sigma(10) \sigma(1) \sigma(2) \sigma(3) \sigma(4)} S_{\sigma(6) \sigma(7) \sigma(8)}} \\
& +\frac{1}{2 s_{\sigma(1) \sigma(2) \sigma(3)} S_{\sigma(1) \sigma(2) \sigma(3) \sigma(4) \sigma(5)} S_{\sigma(6) \sigma(7) \sigma(8)}} \\
& +\frac{1}{2 s_{\sigma(1) \sigma(2) \sigma(3)} S_{\sigma(9) \sigma(10) \sigma(1) \sigma(2) \sigma(3)} S_{\sigma(6) \sigma(7) \sigma(8)}} \\
& \left.+\frac{1}{S_{\sigma(1) \sigma(2) \sigma(3)} S_{\sigma(4) \sigma(5) \sigma(6)} S_{\sigma(7) \sigma(8) \sigma(9)}}\right) .
\end{aligned}
$$

As a byproduct, the boundary term of $A_{0}$ is

$$
\begin{aligned}
B= & A_{s_{123}}+A_{s_{(10) 12}}+\frac{1}{s_{345}} \frac{1}{s_{12345}}\left(\frac{1}{s_{678}}+\frac{1}{s_{789}}+\frac{1}{s_{89(10)}}\right) \\
& +\frac{1}{s_{89(10)}} \frac{1}{s_{34567}}\left(\frac{1}{s_{345}}+\frac{1}{s_{456}}+\frac{1}{s_{567}}\right) .
\end{aligned}
$$




\section{Example 2: pure gauge theory}

Now we move on to color-ordered amplitudes of gluons. The lowest-point amplitudes are three-point MHV and anti-MHV amplitudes, which are given as

$$
\begin{aligned}
& A_{3}\left(1^{-}, 2^{-}, 3^{+}\right)=\frac{\langle 1 \mid 2\rangle^{4}}{\langle 1 \mid 2\rangle\langle 2 \mid 3\rangle\langle 3 \mid 1\rangle}, \\
& A_{3}\left(1^{+}, 2^{+}, 3^{-}\right)=\frac{[1 \mid 2]^{4}}{[1 \mid 2][2 \mid 3][3 \mid 1]},
\end{aligned}
$$

where the coupling constant has been neglected. As is well known, these amplitudes will vanish when $z \rightarrow \infty$ under correct deformations, therefore they can be computed by the BCFW approach [9]. We will use our approach to reproduce them. The results in this section can also be found in [5]. In this section, the calculation will start by computing the factorization limit for one channel.

\subsection{The MHV amplitude $A_{n}\left(1^{+} \ldots i^{-} \cdots j^{-} \cdots n^{+}\right)$}

The first case is the $n$-point MHV amplitude, given by the well known formula

$$
A_{n}\left(1^{+} \cdots i^{-} \cdots j^{-} \cdots n^{+}\right)=\frac{\langle i \mid j\rangle^{4}}{\langle 1 \mid 2\rangle\langle 2 \mid 3\rangle \cdots\langle n-1 \mid n\rangle\langle n \mid 1\rangle} \text {. }
$$

It is sufficient to consider $A_{n}\left(1^{-} \cdots i^{-} \cdots n^{+}\right)$since the general formula can be transformed into this choice by cyclic permutation. We assume (32) is valid for $m$-point MHV amplitudes with $m<n$, then consider factorization limits of the $n$-point MHV amplitude. First, let us consider the limit $s_{12} \rightarrow 0$. There are two types of solutions,

$I_{1}: \lambda_{2}=\alpha \lambda_{1}, \quad P_{12}=\lambda_{1}\left(\tilde{\lambda}_{1}+\alpha \tilde{\lambda}_{2}\right)$,

$I_{2}: \tilde{\lambda}_{2}=\beta \tilde{\lambda}_{1}, \quad P_{12}=\left(\lambda_{1}+\beta \lambda_{2}\right) \tilde{\lambda}_{1}$.

Solution $I_{2}$ contributes nothing to the factorization limit, since no matter which helicity is assigned for the internal propagator, one of the sub-amplitudes $A_{L}$ and $A_{R}$ vanishes, thus only the solution $I_{1}$ is considered. Then ${ }^{4}$

$$
\begin{aligned}
& \lim _{\langle 1 \mid 2\rangle \rightarrow 0} A_{3}\left(1^{-}, 2^{+},-P_{12}^{+}\right) A_{n-1}\left(P_{12}^{-}, 3^{+}, \ldots i^{-} \ldots n^{+}\right) \\
& =\frac{\left[2 \mid-P_{12}\right]^{3}}{\left[-P_{12} \mid 1\right][1 \mid 2]} \frac{\left\langle i \mid P_{12}\right\rangle^{4}}{\langle P \mid 3\rangle\langle 3 \mid 4\rangle \cdots\left\langle n \mid P_{12}\right\rangle} \\
& =\frac{[2 \mid 1]^{3}}{\alpha[2 \mid 1][1 \mid 2]} \frac{\langle i \mid 1\rangle^{4}}{\langle 1 \mid 3\rangle\langle 3 \mid 4\rangle \cdots\langle n \mid 1\rangle} \\
& =\frac{[1 \mid 2]\langle 1 \mid i\rangle^{4}}{\langle 2 \mid 3\rangle\langle 3 \mid 4\rangle \cdots\langle n \mid 1\rangle}
\end{aligned}
$$

\footnotetext{
${ }^{4}$ For the complex momentum $-P$, one can choose corresponding spinors as $\lambda_{\tilde{\lambda}_{P}}=\lambda_{P}$ and $\widetilde{\lambda}_{-P}=-\widetilde{\lambda}_{P}$. In this choice, $\widetilde{\lambda}_{-P}$ can be replaced by $\tilde{\lambda}_{P}$ if it appears for even times.
}

where we have used $\alpha\langle 1 \mid 3\rangle=\langle 2 \mid 3\rangle$. From this we can get the starting expression

$$
A_{\langle 1 \mid 2\rangle}=-\frac{\lim _{\langle 1 \mid 2\rangle \rightarrow 0} A_{L} A_{R}}{s_{12}}=\frac{\langle 1 \mid i\rangle^{4}}{\langle 1 \mid 2\rangle\langle 2 \mid 3\rangle\langle 3 \mid 4\rangle \cdots\langle n \mid 1\rangle} .
$$

Although for this special case it is already the correct result, logically, we still need to check whether it has the correct factorization limits for other channels. For instance, let us consider the limit $s_{(j-1) j} \rightarrow 0$ where both $(j-1)$ and $j$ have positive helicity. The non-vanishing sub-amplitude corresponds to the solution $\lambda_{j}=\alpha \lambda_{j-1}, P_{(j-1) j}=\lambda_{j-1}\left(\tilde{\lambda}_{j-1}+\right.$ $\left.\alpha \widetilde{\lambda}_{j}\right)$. Then we have

$$
\begin{aligned}
\lim _{\langle j-1 \mid j\rangle \rightarrow 0} A_{3}\left((j-1)^{+}, j^{+},-P_{(j-1) j}^{-}\right) A_{n-1} \\
\quad \times\left(P_{(j-1) j}^{+},(j+1)^{+}, \cdots 1^{-} \cdots i^{-} \cdots(j-2)^{+}\right) \\
=\frac{[j-1 \mid j]^{3}}{\left[j \mid-P_{(j-1) j}\right]\left[-P_{(j-1) j \mid j-1]}\right.} \\
\quad \times \frac{\langle 1 \mid i\rangle^{4}}{\left\langle P_{(j-1) j} \mid j+1\right\rangle\langle j+1 \mid j+2\rangle \cdots\left\langle j-2 \mid P_{(j-1) j}\right\rangle} \\
=\frac{[j-1 \mid j]\langle 1 \mid i\rangle^{4}}{\alpha\langle j-1 \mid j+1\rangle\langle j+1 \mid j+2\rangle \cdots\langle j-2 \mid j-1\rangle} \\
=\frac{[j-1 \mid j]\langle 1 \mid i\rangle^{4}}{\langle j \mid j+1\rangle\langle j+1 \mid j+2\rangle \cdots\langle j-2 \mid j-1\rangle} \\
=
\end{aligned}
$$

therefore $A_{\langle 1 \mid 2\rangle}$ provides the correct factorization limit for $s_{(j-1) j} \rightarrow 0$. It can also be checked that $A_{\langle 1 \mid 2\rangle}$ provides the correct factorization limits for the other channels. Therefore we can conclude that $A_{\langle 1 \mid 2\rangle}$ is the amplitude $A_{n}\left(1^{-} \cdots i^{-} \cdots n^{+}\right)$that has all correct factorization limits.

4.2 The six-point amplitude $A_{6}\left(1^{-}, 2^{-}, 3^{-}, 4^{+}, 5^{+}, 6^{+}\right)$

Now we turn to the six-point NMHV amplitude $A_{6}\left(1^{-}, 2^{-}\right.$, $\left.3^{-}, 4^{+}, 5^{+}, 6^{+}\right)$. First let us consider the limit $s_{12} \rightarrow 0$. The solution for the non-vanishing sub-amplitude is

$\tilde{\lambda}_{2}=\alpha \tilde{\lambda}_{1}, \quad P_{12}=\left(\lambda_{1}+\alpha \lambda_{2}\right) \tilde{\lambda}_{1}$,

We use an auxiliary spinor $\eta$ to express the un-determined parameter $\alpha$ as $\alpha=\frac{[\eta \mid 2]}{[\eta \mid 1]}$. Then

$$
\begin{aligned}
A_{[1 \mid 2]} & =-\frac{\lim _{[1 \mid 2] \rightarrow 0} A_{3}\left(1^{-}, 2^{-},-P_{12}^{+}\right) A_{5}\left(P_{12}^{-}, 3^{-}, 4^{+}, 5^{+}, 6^{+}\right)}{s_{12}} \\
& =\frac{1}{[1 \mid 2][\eta \mid 1][2 \mid \eta]} \frac{\langle 3|1+2| \eta]^{3}}{\langle 3 \mid 4\rangle\langle 4 \mid 5\rangle\langle 5 \mid 6\rangle\langle 6|1+2| \eta]}
\end{aligned}
$$

Notice that the spinor $\eta$ can be chosen arbitrarily. It is exactly the ambiguity we have emphasized in Sect. 2. Different choices of $\eta$ gives the same result only under the limit $[1 \mid 2] \rightarrow 0$. Also since there are three pairs of $\eta$ (we count one 
in the numerator and one in the denominator as a pair), each pair can be chosen independently. For the current example, we choose three pairs of $\eta$ to be the same. In other words, we have chosen a type of representative expressions in the category of the limit $[1 \mid 2] \rightarrow 0$.

To fix $\eta$, we can try to choose one value so that $A_{[1 \mid 2]}$ has the correct factorization limits for other channels, thus we pick a pole contained in $A_{[12]}$. Let us consider the limit $s_{45} \rightarrow 0$, the solution corresponding to the non-vanishing sub-amplitudes is

$\lambda_{5}=\alpha \lambda_{4}, \quad P_{45}=\lambda_{4}\left(\tilde{\lambda}_{4}+\alpha \tilde{\lambda}_{5}\right), \quad \alpha=\frac{\langle\zeta \mid 4\rangle}{\langle\zeta \mid 5\rangle}$,

then we have

$$
\begin{aligned}
A_{\langle 4 \mid 5\rangle} & =-\frac{\lim _{\langle 4 \mid 5\rangle \rightarrow 0} A_{3}\left(4^{+}, 5^{+},-P_{45}^{-}\right) A_{5}\left(P_{45}^{+}, 6^{+}, 1^{-}, 2^{-}, 3^{-}\right)}{s_{45}} \\
& =\frac{1}{\langle 4 \mid 5\rangle\langle\zeta \mid 4\rangle\langle 5 \mid \zeta\rangle} \frac{\langle\zeta|4+5| 6]^{3}}{[6 \mid 1][1 \mid 2][2 \mid 3]\langle\zeta|4+5| 3]} .
\end{aligned}
$$

Again, the form of $A_{\langle 4 \mid 5\rangle}$ provides a type of representative expressions in the category of the limit $\langle 4 \mid 5\rangle \rightarrow 0$. Now we ask if there is a choice of $\eta$ and $\zeta$, such that the above two representative expressions are the same under the corresponding limits, i.e., $\left(s_{12} s_{45} A_{[1 \mid 2]}\right)_{[1 \mid 2] \rightarrow 0}=\left(s_{12} s_{45} A_{\langle 4 \mid 5\rangle}\right)_{\langle 4 \mid 5\rangle \rightarrow 0}$. This is a strong constraint, since it means that two correct factorization limits are given either by $A_{[1 \mid 2]}$ or by $A_{\langle 4 \mid 5\rangle}$. In general, it cannot be achieved, but for this case, we fortunately manage to obtain the choice $\eta=\tilde{\lambda}_{6}, \quad \zeta=\lambda_{3}$. To check it, for the left hand side, we have

$$
\begin{aligned}
& \lim _{[1 \mid 2] \rightarrow 0} A_{3}\left(1^{-}, 2^{-},-P_{12}^{+}\right) A_{5}\left(P_{12}^{-}, 3^{-}, 4^{+}, 5^{+}, 6^{+}\right) \\
& =\frac{\langle 1 \mid 2\rangle\langle 3|1+2| 6]^{3}}{\langle 3 \mid 4\rangle\langle 4 \mid 5\rangle\langle 5 \mid 6\rangle[6 \mid 1][2 \mid 6]\langle 6|1+2| 6]} \\
& =\frac{\langle 1 \mid 2\rangle\langle 3|4+5| 6]^{3}}{\langle 3 \mid 4\rangle\langle 4 \mid 5\rangle[6 \mid 1]\langle 5|6| 2]\left(s_{16}+s_{26}\right)} \\
& =\frac{\langle 1 \mid 2\rangle\langle 3|4+5| 6]^{3}}{\langle 3 \mid 4\rangle\langle 4 \mid 5\rangle[1 \mid 6]\langle 5|3+4| 2] s_{126}},
\end{aligned}
$$

where [1|2] $=0$ is used in the last step, thus $s_{16}+s_{26}=$ $s_{12}+s_{16}+s_{26}=s_{126}$ and $\langle 5|3+4| 2]=-\langle 5|6| 2]$. Similarly, for the right hand side we have

$$
\begin{aligned}
& \lim _{\langle 4 \mid 5\rangle \rightarrow 0} A_{3}\left(4^{+}, 5^{+},-P_{45}^{-}\right) A_{5}\left(P_{45}^{+}, 6^{+}, 1^{-}, 2^{-}, 3^{-}\right) \\
& =\frac{[4 \mid 5]\langle 3|4+5| 6]^{3}}{\langle 3 \mid 4\rangle[1 \mid 2][1 \mid 6]\langle 5|3+4| 2] s_{126}},
\end{aligned}
$$

where we have used $\langle 3|4+5| 3]=s_{126}$ and $\langle 53\rangle[2 \mid 3]=$ $-\langle 5|3+4| 2]$ under the limit $\langle 4 \mid 5\rangle \rightarrow 0$.

Now a nice starting expression appears

$$
\begin{aligned}
A_{[1 \mid 2]\langle 4 \mid 5\rangle} & =A_{[1 \mid 2]}=A_{\langle 4 \mid 5\rangle} \\
& =-\frac{\langle 3|4+5| 6]^{3}}{\langle 3 \mid 4\rangle\langle 4 \mid 5\rangle[1 \mid 2][6 \mid 1]\langle 5|3+4| 2] s_{126}} .
\end{aligned}
$$

To continue, we consider other poles. One nice choice is a pole $s$ such that $\lim _{s \rightarrow 0} s A_{[1 \mid 2]\langle 4 \mid 5\rangle}=0$. There are two two-particle channels $s_{23} \rightarrow 0$ and $s_{56} \rightarrow 0$ satisfying this requirement. Proceeding as the case [1|2] $\rightarrow 0$ and $\langle 4 \mid 5\rangle \rightarrow$ 0 , we get

$$
A_{[2 \mid 3]|5| 6\rangle}=-\frac{\langle 1|2+3| 4]^{3}}{\langle 6 \mid 1\rangle\langle 5 \mid 6\rangle[2 \mid 3][3 \mid 4]\langle 5|3+4| 2] s_{234}},
$$

which has the correct factorization limits for $s_{23} \rightarrow 0$ and $s_{56} \rightarrow 0$. Since $A_{[1 \mid 2]\langle 4 \mid 5\rangle}$ and $A_{[2 \mid 3]\langle 5 \mid 6\rangle}$ do not share any physical pole, we should sum them to get

$$
\begin{aligned}
A_{[1 \mid 2][2 \mid 3]\langle 4 \mid 5\rangle\langle 5 \mid 6\rangle}=A_{[1 \mid 2]\langle 4 \mid 5\rangle}+A_{[2 \mid 3]|5| 6\rangle} \\
=-\frac{1}{\langle 5|3+4| 2]}\left(\frac{\langle 1|2+3| 4]^{3}}{\langle 6 \mid 1\rangle\langle 5 \mid 6\rangle[2 \mid 3][3 \mid 4] s_{234}}\right. \\
\left.+\frac{\langle 3|4+5| 6]^{3}}{\langle 3 \mid 4\rangle\langle 4 \mid 5\rangle[1 \mid 2][6 \mid 1] s_{126}}\right),
\end{aligned}
$$

which gives the correct factorization limits for $s_{12} \rightarrow 0$, $s_{23} \rightarrow 0, s_{45} \rightarrow 0$, and $s_{56} \rightarrow 0$. One can verify that $A_{[1 \mid 2][2 \mid 3]\langle 4 \mid 5\rangle\langle 5 \mid 6\rangle}$ also gives the correct factorization limits for remaining channels. ${ }^{5}$

One can observe the factor $\langle 5|3+4| 2]$ in the denominator of $A_{[1 \mid 2][2 \mid 3]\langle 4 \mid 5\rangle\langle 5 \mid 6\rangle}$. However, it is not a pole since

$$
\lim _{\langle 5|3+4| 2] \rightarrow 0}\langle 5|3+4| 2] A_{[1 \mid 2][2 \mid 3]\langle 4 \mid 5\rangle\langle 5 \mid 6\rangle}=0 .
$$

To verify this, notice that $\langle 5|3+4| 2] \rightarrow 0$ implies $|5\rangle \propto \mid 3+$ $4 \mid 2]$. Then the momentum conservation condition becomes

$$
\begin{aligned}
& |1\rangle[1|+| 2\rangle[2|+| 3\rangle[3|+| 4\rangle[4|+c| 3 \\
& \quad+4 \mid 2][5|+| 6\rangle[6 \mid=0 .
\end{aligned}
$$

The coefficient $c$ can be fixed by contract (47) with two spinors. For example, contracting (47) with $\mid 1]$ and $\langle 6|$, we get $c=-\frac{\langle 1|2+3+4| 6]}{\langle 1|3+4| 2|[5 \mid 6]}$. Contracting with different spinors gives different expressions of $c$ but they are equivalent under the limit $\langle 5|3+4| 2] \rightarrow 0 .{ }^{6}$ Substituting these into (45), one can get the result (46). Consequently, the expression (45) contains only physical poles. Thus, we have found

$$
A_{6}\left(1^{-}, 2^{-}, 3^{-}, 4^{+}, 5^{+}, 6^{+}\right)=A_{[1 \mid 2][2 \mid 3]\langle 4 \mid 5\rangle\langle 5 \mid 6\rangle} \text {. }
$$

Although in this subsection, we start with the factorization of a two-particle channel, one can start with the factorization of a three-particle channel and proceed similarly to get the correct result. The calculation is shown in Appendix B.

\footnotetext{
5 There is a technical issue regarding the limits, such as $s_{234} \rightarrow 0$. For this case, the spinor $\lambda_{P_{234}}$ can be expressed, for example, via $\left\langle a \mid P_{234}\right\rangle=$ $\frac{\left\langle a\left|P_{234}\right| b\right]}{\left[P_{234} \mid b\right]}$ (see Appendix B).

${ }^{6}$ If one in this way fixes the coefficient $\alpha$ in (37), contracting the momentum conservation equation with different spinors, indeed this provides different choices of the reference spinor $\eta$. There is no guidance to show which choice is more proper for the latter calculation.
} 
4.3 The six-point amplitude $A_{6}\left(1^{+}, 2^{-}, 3^{+}, 4^{-}, 5^{+}, 6^{-}\right)$

Let us start with the factorization limit for $s_{12} \rightarrow 0$. There are two types of solutions for non-vanishing sub-amplitudes:

$I_{1}: \lambda_{2}=\alpha_{1} \lambda_{1}, \quad P_{12}=\lambda_{1}\left(\tilde{\lambda}_{1}+\alpha_{1} \tilde{\lambda}_{2}\right), \quad \alpha_{1}=\frac{\left\langle\eta_{1} \mid 2\right\rangle}{\left\langle\eta_{1} \mid 1\right\rangle}$,

$I_{2}: \tilde{\lambda}_{2}=\beta_{1} \tilde{\lambda}_{1}, \quad P_{12}=\left(\lambda_{1}+\beta_{1} \lambda_{2}\right) \tilde{\lambda}_{1}, \quad \beta_{1}=\frac{\left[\zeta_{1} \mid 2\right]}{\left[\zeta_{1} \mid 1\right]}$.

For solution $I_{1}$, we have

$A_{\langle 1 \mid 2\rangle}=\frac{\left\langle 2 \mid \eta_{1}\right\rangle^{3}}{\left\langle\eta_{1} \mid 1\right\rangle\langle 1 \mid 2\rangle} \frac{[3 \mid 5]^{4}}{[3 \mid 4][4 \mid 5][5 \mid 6]\left\langle\eta_{1}|1+2| 6\right]\left\langle\eta_{1}|1+2| 3\right]}$,

and for solution $I_{2}$,

$$
A_{[1 \mid 2]}=\frac{\left[1 \mid \zeta_{1}\right]^{3}\langle 4 \mid 6\rangle^{4}}{\left[2 \mid \zeta_{1}\right][2 \mid 1]\langle 3 \mid 4\rangle\langle 4 \mid 5\rangle\langle 5 \mid 6\rangle\left\langle 6|1+2| \zeta_{1}\right]\left\langle 3|1+2| \zeta_{1}\right]} .
$$

$A_{\langle 1 \mid 2\rangle}$ does not contain the pole [1|2] and $A_{[1 \mid 2]}$ does not contain the pole $\langle 1 \mid 2\rangle$. Thus we sum these two parts to obtain the starting expression $A_{\langle 1 \mid 2\rangle[1 \mid 2]}=A_{\langle 1 \mid 2\rangle}+A_{[1 \mid 2]}$, which satisfies the factorization limit for $s_{12} \rightarrow 0$.

In the expression of $A_{\langle 1 \mid 2\rangle[1 \mid 2]}$, there are unfixed variables $\zeta_{1}$ and $\eta_{1}$, which reflects the freedom of representative elements in the category as discussed in Sect. 2. Now we try to fix these parameters as previous. To do so, considering the limit $s_{23} \rightarrow 0$, a similar calculation leads to

$$
\begin{aligned}
s_{23} \rightarrow 0 & : \\
A_{\langle 2 \mid 3\rangle} & =\frac{\left\langle 2 \mid \eta_{2}\right\rangle^{3}[5 \mid 1]^{4}}{\left\langle 3 \mid \eta_{2}\right\rangle\langle 3 \mid 2\rangle[4 \mid 5][5 \mid 6][6 \mid 1]\left\langle\eta_{2}|2+3| 1\right]\left\langle\eta_{2}|2+3| 4\right]}, \\
A_{[2 \mid 3]} & =\frac{\left[3 \mid \zeta_{2}\right]^{3}\langle 4 \mid 6\rangle^{4}}{\left[\zeta_{2} \mid 2\right][3 \mid 2]\langle 4 \mid 5\rangle\langle 5 \mid 6\rangle\langle 6 \mid 1\rangle\left\langle 1|2+3| \zeta_{2}\right]\left\langle 4|2+3| \zeta_{2}\right]} .
\end{aligned}
$$

Matching $A_{[2 \mid 3]}$ with $A_{[1 \mid 2]}$ yields

$\zeta_{1}=\tilde{\lambda}_{3}, \quad \zeta_{2}=\tilde{\lambda}_{1}$

and

$$
\begin{aligned}
A_{[1 \mid 2][2 \mid 3]} & =A_{[1 \mid 2]}=A_{[2 \mid 3]} \\
& =\frac{[1 \mid 3]^{4}\langle 4 \mid 6\rangle^{4}}{\langle 4 \mid 5\rangle\langle 5 \mid 6\rangle[1 \mid 2][2 \mid 3]\langle 6|1+2| 3]\langle 4|2+3| 1] s_{123}} .
\end{aligned}
$$

Plugging this back, the starting expression becomes $A_{\langle 1 \mid 2\rangle[1 \mid 2][2 \mid 3]}=A_{\langle 1 \mid 2\rangle}+A_{[1 \mid 2][2 \mid 3]}$ which has the correct factorization limits for $s_{12} \rightarrow 0$ plus [2|3] $\rightarrow 0$. When we do the iterative step to include the new factorization limit $\langle 2 \mid 3\rangle \rightarrow 0$, we need to add $A_{\langle 2 \mid 3\rangle}$ to arrive at $A_{\langle 1 \mid 2\rangle\langle 2 \mid 3\rangle[1 \mid 2][2 \mid 3]}=A_{\langle 1 \mid 2\rangle}+A_{\langle 2 \mid 3\rangle}+A_{[1 \mid 2][2 \mid 3]}$, which has the correct factorization limits for $s_{12} \rightarrow 0$ and $s_{23} \rightarrow 0$.
Now we continue to include new factorization limits for $s_{34} \rightarrow 0$. Using

$$
\begin{aligned}
s_{34} \rightarrow 0 & : \\
A_{\langle 3 \mid 4\rangle} & =\frac{\left\langle 4 \mid \eta_{3}\right\rangle^{3}}{\left\langle\eta_{3} \mid 3\right\rangle\langle 3 \mid 4\rangle} \frac{[5 \mid 1]^{4}}{[5 \mid 6][6 \mid 1][1 \mid 2]\left\langle\eta_{3}|3+4| 2\right]\left\langle\eta_{3}|3+4| 5\right]}, \\
A_{[3 \mid 4]} & =\frac{\left[3 \mid \zeta_{3}\right]^{3}\langle 6 \mid 2\rangle^{4}}{\left[4 \mid \zeta_{3}\right][4 \mid 3]\langle 5 \mid 6\rangle\langle 6 \mid 1\rangle\langle 1 \mid 2\rangle\left\langle 2|3+4| \zeta_{3}\right]\left\langle 5|3+4| \zeta_{3}\right]},
\end{aligned}
$$

we find that the freedom of $A_{\langle 2 \mid 3\rangle}$ can be fixed by $\eta_{2}=\lambda_{4}$ when we match it with $A_{\langle 3 \mid 4\rangle}$ with $\eta_{3}=\lambda_{2}$, and this single expression is

$$
\begin{aligned}
& A_{\langle 2 \mid 3\rangle\langle 3 \mid 4\rangle}=A_{\langle 2 \mid 3\rangle}=A_{\langle 3 \mid 4\rangle} \\
& =\frac{\langle 2 \mid 4\rangle^{4}[5 \mid 1]^{4}}{\langle 2 \mid 3\rangle\langle 3 \mid 4\rangle[5 \mid 6][6 \mid 1]\langle 4|2+3| 1]\langle 2|3+4| 5] s_{234}} .
\end{aligned}
$$

Similarly, matching $A_{[3 \mid 4]}$ and $A_{\langle 1 \mid 2\rangle}$ we find $\eta_{1}=\lambda_{6}, \zeta_{3}=$ $\tilde{\lambda}_{5}$ and

$$
\begin{aligned}
& A_{\langle 1 \mid 2\rangle[3 \mid 4]}=A_{\langle 1 \mid 2\rangle}=A_{[3 \mid 4]} \\
& =\frac{\langle 2 \mid 6\rangle^{4}[3 \mid 5]^{4}}{\langle 6 \mid 1\rangle\langle 1 \mid 2\rangle[3 \mid 4][4 \mid 5]\langle 6|4+5| 3]\langle 2|3+4| 5] s_{345}} .
\end{aligned}
$$

Summing (54), (56), and (57) we have

$A_{\langle 1 \mid 2\rangle\langle 2 \mid 3\rangle\langle 3 \mid 4\rangle[1 \mid 2][2 \mid 3][3 \mid 4]}$

$$
\begin{aligned}
= & A_{[1 \mid 2][2 \mid 3]}+A_{\langle 2 \mid 3\rangle\langle 3 \mid 4\rangle}+A_{\langle 1 \mid 2\rangle[3 \mid 4]} \\
= & \frac{[1 \mid 3]^{4}\langle 4 \mid 6\rangle^{4}}{[1 \mid 2][2 \mid 3]\langle 4 \mid 5\rangle\langle 5 \mid 6\rangle\langle 6|1+2| 3]\langle 4|2+3| 1] s_{123}} \\
& +\frac{\langle 2 \mid 4\rangle^{4}[5 \mid 1]^{4}}{\langle 2 \mid 3\rangle\langle 3 \mid 4\rangle[5 \mid 6][6 \mid 1]\langle 4|2+3| 1]\langle 2|3+4| 5] s_{234}} \\
& +\frac{\langle 2 \mid 6\rangle^{4}[3 \mid 5]^{4}}{\langle 6 \mid 1\rangle\langle 1 \mid 2\rangle[3 \mid 4][4 \mid 5]\langle 6|4+5| 3]\langle 2|3+4| 5] s_{345}} .
\end{aligned}
$$

One can check that $A_{\langle 1 \mid 2\rangle\langle 2 \mid 3\rangle\langle 3 \mid 4\rangle[1 \mid 2][2 \mid 3][3 \mid 4]}$ has the correct factorization limits for all channels. Again, it can be verified that there is no spurious pole. Thus we find

$A_{6}\left(1^{+}, 2^{-}, 3^{+}, 4^{-}, 5^{+}, 6^{-}\right)=A_{\langle 1 \mid 2\rangle\langle 2 \mid 3\rangle\langle 3 \mid 4\rangle[1 \mid 2][2 \mid 3][3 \mid 4]}$.

4.4 The six-point amplitude $A_{6}\left(1^{+}, 2^{+}, 3^{-}, 4^{+}, 5^{-}, 6^{-}\right)$

Let us start with the factorization limit of $s_{12} \rightarrow 0$. There is only one type of solution corresponding to the non-vanishing sub-amplitude, namely $\lambda_{2}=\alpha \lambda_{1}$, which leads to

$A_{\langle 1 \mid 2\rangle}=\frac{\left\langle\eta_{1}|1+2| 4\right]^{4}}{\langle 2 \mid 1\rangle\left\langle 2 \mid \eta_{1}\right\rangle\left\langle 1 \mid \eta_{1}\right\rangle[3 \mid 4][4 \mid 5][5 \mid 6]\left\langle\eta_{1}|1+2| 3\right]\left\langle\eta_{1}|1+2| 6\right]}$. 
To fix the choice of $\eta_{1}$, noticing the pole [5|6] in $A_{\langle 1 \mid 2\rangle}$, we then match it with the factorization limit,

$$
A_{[5 \mid 6]}=\frac{\left\langle 3|5+6| \zeta_{1}\right]^{4}}{[6 \mid 5]\left[\zeta_{1} \mid 5\right]\left[\zeta_{1} \mid 6\right]\langle 1 \mid 2\rangle\langle 2 \mid 3\rangle\langle 3 \mid 4\rangle\left\langle 1|5+6| \zeta_{1}\right]\left\langle 4|5+6| \zeta_{1}\right]},
$$

thus we find $\eta_{1}=\lambda_{3}, \quad \zeta_{1}=\tilde{\lambda}_{4}$, and the starting expression is given by

$$
\begin{aligned}
& A_{\langle 1 \mid 2\rangle[5 \mid 6]}=A_{\langle 1 \mid 2\rangle}=A_{[5 \mid 6]} \\
& =-\frac{\langle 3|1+2| 4]^{4}}{\langle 1 \mid 2\rangle\langle 2 \mid 3\rangle[4 \mid 5][5 \mid 6]\langle 1|2+3| 4]\langle 3|1+2| 6] s_{123}} .
\end{aligned}
$$

Since $A_{\langle 1 \mid 2\rangle[5 \mid 6]}$ does not contain the poles $s_{34}$ and $s_{61}$, we will try to include their factorization limits. The limit $s_{34} \rightarrow 0$ gives

$$
\begin{aligned}
A_{\langle 3 \mid 4\rangle} & =\frac{\left\langle 3 \mid \eta_{2}\right\rangle^{3}[1 \mid 2]^{3}}{\langle 4 \mid 3\rangle\left\langle 4 \mid \eta_{2}\right\rangle[5 \mid 6][6 \mid 1]\left\langle\eta_{2}|3+4| 2\right]\left\langle\eta_{2}|3+4| 5\right]}, \\
A_{[3 \mid 4]} & =\frac{\langle 5 \mid 6\rangle^{3}\left[\zeta_{2} \mid 4\right]^{3}}{[4 \mid 3]\left[\zeta_{2} \mid 3\right]\langle 6 \mid 1\rangle\langle 1 \mid 2\rangle\left\langle 2|3+4| \zeta_{2}\right]\left\langle 5|3+4| \zeta_{2}\right]},
\end{aligned}
$$

while the limit $s_{61} \rightarrow 0$ gives

$$
\begin{aligned}
A_{\langle 6 \mid 1\rangle} & =\frac{\left\langle\eta_{3} \mid 6\right\rangle^{3}[2 \mid 4]^{4}}{\langle 1 \mid 6\rangle\left\langle 1 \mid \eta_{3}\right\rangle[2 \mid 3][3 \mid 4][4 \mid 5]\left\langle\eta_{3}|1+6| 5\right]\left\langle\eta_{3}|1+6| 2\right]}, \\
A_{[6 \mid 1]} & =\frac{\langle 3 \mid 5\rangle^{4}\left[1 \mid \zeta_{3}\right]^{4}}{[1 \mid 6]\left[6 \mid \zeta_{3}\right]\langle 2 \mid 3\rangle\langle 3 \mid 4\rangle\langle 4 \mid 5\rangle\left\langle 5|6+1| \zeta_{3}\right]\left\langle 2|6+1| \zeta_{3}\right]} .
\end{aligned}
$$

Matching $A_{[6 \mid 1]}$ with $A_{\langle 3 \mid 4\rangle}$ fixes $\eta_{2}=\lambda_{5}, \quad \zeta_{3}=\tilde{\lambda}_{2}$, and we get

$$
\begin{aligned}
A_{\langle 3 \mid 4\rangle[6 \mid 1]} & =A_{\langle 3 \mid 4\rangle}=A_{[6 \mid 1]} \\
& =-\frac{\langle 3 \mid 5\rangle^{4}[1 \mid 2]^{3}}{\langle 3 \mid 4\rangle\langle 4 \mid 5\rangle[6 \mid 1]\langle 5|3+4| 2]\langle 3|4+5| 6] s_{345}} .
\end{aligned}
$$

Then matching $A_{\langle 6 \mid 1\rangle}$ with $A_{[3 \mid 4]}$ fixes $\eta_{3}=\lambda_{5}, \zeta_{2}=\tilde{\lambda}_{2}$, and

$$
\begin{aligned}
A_{\langle 6 \mid 1\rangle[3 \mid 4]} & =A_{\langle 6 \mid 1\rangle}=A_{[3 \mid 4]} \\
& =-\frac{\langle 5 \mid 6\rangle^{3}[2 \mid 4]^{4}}{\langle 6 \mid 1\rangle[2 \mid 3][3 \mid 4]\langle 1|2+3| 4]\langle 5|3+4| 2] s_{234}} .
\end{aligned}
$$

Summing these, we have

$$
\begin{aligned}
& A_{\langle 1 \mid 2\rangle\langle 3 \mid 4\rangle\langle 6 \mid 1\rangle[3 \mid 4][5 \mid 6][6 \mid 1]} \\
& =A_{\langle 1 \mid 2\rangle[5 \mid 6]}+A_{\langle 3 \mid 4\rangle[6 \mid 1]}+A_{\langle 6 \mid 1\rangle[3 \mid 4]} \\
& =-\frac{\langle 3|1+2| 4]^{4}}{\langle 1 \mid 2\rangle\langle 2 \mid 3\rangle[4 \mid 5][5 \mid 6]\langle 1|2+3| 4]\langle 3|1+2| 6] s_{123}} \\
& \quad-\frac{\langle 3 \mid 5\rangle^{4}[1 \mid 2]^{3}}{[6 \mid 1]\langle 3 \mid 4\rangle\langle 4 \mid 5\rangle\langle 5|3+4| 2]\langle 3|4+5| 6] s_{345}} \\
& \quad-\frac{\langle 5 \mid 6\rangle^{3}[2 \mid 4]^{4}}{[2 \mid 3][3 \mid 4]\langle 6 \mid 1\rangle\langle 1|2+3| 4]\langle 5|3+4| 2] s_{234}} .
\end{aligned}
$$

It can be verified that $A_{\langle 1 \mid 2\rangle\langle 3 \mid 4\rangle\langle 6 \mid 1\rangle[3 \mid 4][5 \mid 6][6 \mid 1]}$ has the correct factorization limits for all channels, and all spurious poles are canceled, therefore

$A_{6}\left(1^{+}, 2^{+}, 3^{-}, 4^{+}, 5^{-}, 6^{-}\right)=A_{\langle 1 \mid 2\rangle\langle 3 \mid 4\rangle\langle 6 \mid 1\rangle[3 \mid 4][5 \mid 6][6 \mid 1] .}$.

\section{Example 3: Einstein-Maxwell theory}

In this section we consider amplitudes of photons coupling to gravitons. In such a theory the lowest-point amplitudes are of two types: photon-photon-graviton and three-graviton selfinteractions,

$$
\begin{aligned}
& A_{3}\left(a_{\gamma}^{-1}, b_{\gamma}^{+1}, c_{g}^{-2}\right)=\kappa \frac{\langle c \mid a\rangle^{4}}{\langle a \mid b\rangle^{2}}, \\
& A_{3}\left(a_{\gamma}^{-1}, b_{\gamma}^{+1}, c_{g}^{+2}\right)=\kappa \frac{[b \mid c]^{4}}{[a \mid b]^{2}}, \\
& A_{3}\left(a_{g}^{-2}, b_{g}^{-2}, c_{g}^{+2}\right)=\kappa \frac{\langle a \mid b\rangle^{8}}{\langle a \mid b\rangle^{2}\langle b \mid c\rangle^{2}\langle c \mid a\rangle^{2}}, \\
& A_{3}\left(a_{g}^{+2}, b_{g}^{+2}, c_{g}^{-2}\right)=\kappa \frac{[a \mid b]^{8}}{[a \mid b]^{2}[b \mid c]^{2}[c \mid a]^{2}} .
\end{aligned}
$$

Since two types of three-point amplitudes have the same coupling constant $\kappa$, we will neglect $\kappa$ from now on.

Before starting the calculation, let us give a brief review on some general properties of amplitudes in this theory. First, let us review the validity of the BCFW approach. As proved by Cheung, for amplitudes containing at least one graviton, the boundary term is zero under some proper deformations [10]. Hence, the BCFW approach is available for amplitudes containing gravitons. On the other hand, Arkani-Hamed and Kaplan have shown that the boundary term will not vanish when deforming two photons [9]. However, their conclusion cannot be applied to the circumstance that two deformed photons have the same helicity. The reason is that their approach relies on the picture that a hard photon moves in a soft background, which means two deformed photons should be connected by photon propagators or they are attached to the same vertex. In Einstein-Maxwell theory, two photons with the same helicity could not satisfy such a condition because of the helicity structure of three-point amplitudes. In some cases, the naive power counting of individual Feynman diagrams shows $A(z \rightarrow \infty)=0$ when deforming two photons with the same helicity, for example the four-point amplitude $A_{4}\left(1_{\gamma}^{-1}, 2_{\gamma}^{+1}, 3_{\gamma}^{-1}, 4_{\gamma}^{+1}\right)$, then the BCFW approach is feasible. However, if an amplitude contains no graviton and the naive analysis of Feynman diagrams cannot guarantee that it will vanish at $z \rightarrow \infty$, we do not know whether it can be computed by the BCFW approach. We will see such an example, namely $A_{6}\left(1_{\gamma}^{-1}, 2_{\gamma}^{+1}, 3_{\gamma}^{-1}, 4_{\gamma}^{+1}, 5_{\gamma}^{-1}, 6_{\gamma}^{+1}\right)$. Secondly, amplitudes of this theory do not have color-order. Thus, the only difference between external particles which have the same 
helicity is their momenta. It means that amplitudes are invariant when exchanging labels of particles with the same helicity. This symmetry is useful for calculating and checking results.

The structure of three-point amplitudes indicates that an amplitude of this theory must contain even number of photons, and the sum of helicities of photons is zero. Thus, if we focus on amplitudes containing photons, there are two types of four-point amplitudes, two types of five-point amplitudes, three types of six-point amplitudes, and so on. We will calculate all four-point amplitudes, all five-point amplitudes, and one type of six-point amplitudes, namely $A_{6}\left(1_{\gamma}^{-1}, 2_{\gamma}^{+1}, 3_{\gamma}^{-1}, 4_{\gamma}^{+1}, 5_{\gamma}^{-1}, 6_{\gamma}^{+1}\right)$. For four-point and fivepoint amplitudes, we will present more tricks to fix formulas of factorization limits by considering the consistency between different channels. On the other hand, for the sixphoton amplitude, we will discuss how to handle one situation: Among all possible deformations, we know the boundary term will appear for some deformations, and we do not know whether the boundary term will vanish for other deformations.

\subsection{The four-point amplitude $A_{4}\left(1_{\gamma}^{-1}, 2_{\gamma}^{+1}, 3_{g}^{-2}, 4_{g}^{+2}\right)$}

First let us consider $A_{4}\left(1_{\gamma}^{-1}, 2_{\gamma}^{+1}, 3_{g}^{-2}, 4_{g}^{+2}\right)$. We start from the factorization limit of $s_{12} \rightarrow 0$, and there are two types of solutions,

$$
\begin{aligned}
I_{1}: \tilde{\lambda}_{2} & =\alpha \tilde{\lambda}_{1}, \quad \lambda_{3}=\beta \lambda_{4}, \quad P_{12}=\gamma \lambda_{4} \tilde{\lambda}_{1}, \\
\alpha & =\frac{\langle 4 \mid 1\rangle}{\langle 2 \mid 4\rangle}, \quad \beta=\frac{[1 \mid 4]}{[3 \mid 1]}, \quad \gamma=\frac{\langle 1 \mid 2\rangle}{\langle 4 \mid 2\rangle}=\frac{[3 \mid 4]}{[1 \mid 3]}, \\
I_{2}: \lambda_{2} & =\alpha \lambda_{1}, \quad \tilde{\lambda}_{3}=\beta \widetilde{\lambda}_{4}, \quad P_{12}=\gamma \lambda_{1} \tilde{\lambda}_{4}, \\
\alpha & =\frac{[4 \mid 1]}{[2 \mid 4]}, \quad \beta=\frac{\langle 1 \mid 4\rangle}{\langle 3 \mid 1\rangle}, \quad \gamma=\frac{[1 \mid 2]}{[4 \mid 2]}=\frac{\langle 3 \mid 4\rangle}{\langle 1 \mid 3\rangle} .
\end{aligned}
$$

For solution $I_{1}$, we have

$$
\begin{aligned}
& \lim _{[1 \mid 2] \rightarrow 0} A_{3}\left(1_{\gamma}^{-1}, 2_{\gamma}^{+1},-P_{g}^{-2}\right) A_{3}\left(P_{g}^{+2}, 3_{g}^{-2}, 4_{g}^{+2}\right) \\
& =\frac{\langle 1 \mid 3\rangle^{2}\langle 2 \mid 3\rangle^{2}[4 \mid 2]^{4}}{s_{13}^{2}},
\end{aligned}
$$

and for solution $I_{2}$

$$
\begin{aligned}
& \lim _{\langle 12\rangle \rightarrow 0} A_{3}\left(1_{\gamma}^{-1}, 2_{\gamma}^{+1},-P_{g}^{+2}\right) A_{3}\left(P_{g}^{-2}, 3_{g}^{-2}, 4_{g}^{+2}\right) \\
& =\frac{\langle 1 \mid 3\rangle^{2}\langle 2 \mid 3\rangle^{2}[4 \mid 2]^{4}}{s_{14}^{2}} .
\end{aligned}
$$

Now we want to seek a formula $A_{\langle 1 \mid 2\rangle[1 \mid 2]}$ which satisfies factorization limits for both $\langle 1 \mid 2\rangle \rightarrow 0$ and [1|2] $\rightarrow 0$. It can be done by rewriting two factorization limits in the correct expressions. This technique will be used frequently in the latter examples. A useful observation is $s_{13}=-s_{14}$ when $s_{12} \rightarrow 0$, since $s_{12}+s_{13}+s_{14}=0$ for massless particles.
Using this relation, we have

$$
\lim _{\langle 1 \mid 2\rangle \rightarrow 0} A_{L} A_{R}=\lim _{[1 \mid 2] \rightarrow 0} A_{L} A_{R}=-\frac{\langle 1 \mid 3\rangle^{2}\langle 2 \mid 3\rangle^{2}[4 \mid 2]^{4}}{s_{13} s_{14}} .
$$

Thus we can write

$A_{\langle 1 \mid 2\rangle[1 \mid 2]}=\frac{\langle 1 \mid 3\rangle^{2}\langle 2 \mid 3\rangle^{2}[4 \mid 2]^{4}}{s_{12} s_{13} s_{14}}$.

One can check that $A_{\langle 1 \mid 2\rangle[1 \mid 2]}$ has the correct factorization limits for the remaining channels, therefore it is the correct result of $A_{4}\left(1_{\gamma}^{-1}, 2_{\gamma}^{+1}, 3_{g}^{-2}, 4_{g}^{+2}\right)$. It is the same as the one in [16].

\subsection{The four-point amplitude $A_{4}\left(1_{\gamma}^{-1}, 2_{\gamma}^{+1}, 3_{\gamma}^{-1}, 4_{\gamma}^{+1}\right)$}

The second amplitude is $A_{4}\left(1_{\gamma}^{-1}, 2_{\gamma}^{+1}, 3{ }_{\gamma}^{-1}, 4_{\gamma}^{+1}\right)$. Notice that the naive power counting of Feynman diagrams shows $A(z \rightarrow \infty) \rightarrow \frac{1}{z}$ under the deformation of two photons of the same helicity, thus the BCFW approach is feasible although this case cannot be covered by the conclusions in $[9,10]$. Here we try to find the amplitude using our approach. We will start from considering the limit $s_{12} \rightarrow 0$, where two types of solutions are the same as in the previous example and we find

$A_{[1 \mid 2]}=-\frac{\langle 1 \mid 3\rangle^{2}[2 \mid 4]^{2}}{s_{12}}, \quad A_{\langle 1 \mid 2\rangle}=-\frac{\langle 1 \mid 3\rangle^{2}[2 \mid 4]^{2}}{s_{12}}$.

So we can write $A_{\langle 1 \mid 2\rangle[1 \mid 2]}=A_{\langle 1 \mid 2\rangle}=A_{[1 \mid 2]}$ as our starting expression.

Since $A_{\langle 1 \mid 2\rangle[1 \mid 2]}$ does not contain the pole $s_{14}$, we need to add a new term to get the full answer. The limit $s_{14} \rightarrow 0$ gives

$A_{\langle 1 \mid 4\rangle[1 \mid 4]}=-\frac{\langle 1 \mid 3\rangle^{2}[2 \mid 4]^{2}}{s_{14}}$.

Finally we sum $A_{\langle 1 \mid 2\rangle[1 \mid 2]}$ and $A_{\langle 1 \mid 4\rangle[1 \mid 4]}$ to get

$$
\begin{aligned}
A_{\langle 1 \mid 2\rangle\langle 1 \mid 4\rangle[1 \mid 2][1 \mid 4]} & =A_{\langle 1 \mid 2\rangle[1 \mid 2]}+A_{\langle 1 \mid 4\rangle[1 \mid 4]} \\
& =\frac{s_{13}\langle 1 \mid 3\rangle^{2}[2 \mid 4]^{2}}{s_{12} s_{14}}
\end{aligned}
$$

which has the correct factorization limits for $s_{12} \rightarrow 0$ and $s_{14} \rightarrow 0$. It can be verified that $A_{\langle 1 \mid 2\rangle\langle 1 \mid 4\rangle[1 \mid 2][1 \mid 4]}$ also has the correct factorization limits for remain channels. Thus $A_{\langle 1 \mid 2\rangle\langle 1 \mid 4\rangle[1 \mid 2][1 \mid 4]}$ is the correct result for the amplitude $A_{4}\left(1_{\gamma}^{-1}, 2_{\gamma}^{+1}, 3_{\gamma}^{-1}, 4_{\gamma}^{+1}\right)$. It is the same as the one in [16].

5.3 The five-point amplitude $A_{5}\left(1_{\gamma}^{-1}, 2_{\gamma}^{+1}, 3_{g}^{-2}, 4_{g}^{+2}, 5_{g}^{-2}\right)$

Now we turn to the five-point amplitude $A_{5}\left(1_{\gamma}^{-1}, 2_{\gamma}^{+1}, 3_{g}^{-2}\right.$, $\left.4_{g}^{+2}, 5_{g}^{-2}\right)$. Brief analysis of sub-amplitudes shows that this amplitude only contains poles of the form $[\bullet \mid \bullet]$, therefore we need not to consider any channel of the form $\langle\bullet \mid \bullet\rangle$. We start 
with the result of the BCFW approach through a deformation which yields the non-zero boundary term. If we deform $1^{-1}$ and $2^{+1}$, the conclusion in [9] indicates that the boundary contribution will appear. Under the deformation

$\lambda_{2} \rightarrow \lambda_{2}-z \lambda_{1}, \quad \tilde{\lambda}_{1} \rightarrow \tilde{\lambda}_{1}+z \tilde{\lambda}_{2}$,

the BCFW approach gives

$$
\begin{aligned}
A_{[1 \mid 3][1 \mid 5]}= & \frac{\langle 1 \mid 3\rangle\langle 4 \mid 5\rangle[3 \mid 4][2 \mid 4]^{5}}{[1 \mid 3][2 \mid 5][3 \mid 5][4 \mid 5][2 \mid 3]^{2}} \\
& +\frac{\langle 1 \mid 5\rangle\langle 3 \mid 4\rangle[4 \mid 5][2 \mid 4]^{5}}{[1 \mid 5][2 \mid 3][3 \mid 4][3 \mid 5][2 \mid 5]^{2}},
\end{aligned}
$$

which has the correct factorization limits for the poles [1|3] and [1|5]. The expression (79) is just one representation in the category and we can deform it to another while keeping the correct factorization limits for the poles [1|3] and [1|5]. The reason to choose another representation is that

$$
\lim _{[2 \mid 5] \rightarrow 0} s_{25} A_{[1 \mid 3][1 \mid 5]}=\infty, \quad \lim _{[2 \mid 3] \rightarrow 0} s_{23} A_{[1 \mid 3][1 \mid 5]}=\infty .
$$

To remove double poles, we can use following transformations:

$$
\begin{aligned}
\lim _{[1 \mid 3] \rightarrow 0} s_{13} A_{[1 \mid 3][1 \mid 5]} & =-\frac{\langle 1 \mid 3\rangle^{2}\langle 4 \mid 5\rangle[3 \mid 4][2 \mid 4]^{5}}{[2 \mid 5][3 \mid 5][4 \mid 5][2 \mid 3]^{2}} \\
& =\frac{\langle 1 \mid 3\rangle^{2}\langle 2 \mid 5\rangle[3 \mid 2][2 \mid 4]^{5}}{[2 \mid 5][3 \mid 5][4 \mid 5][2 \mid 3]^{2}} \\
& =-\frac{\langle 1 \mid 3\rangle^{2}\langle 2 \mid 5\rangle[2 \mid 4]^{5}}{[2 \mid 3][2 \mid 5][3 \mid 5][4 \mid 5]}
\end{aligned}
$$

and

$$
\begin{aligned}
\lim _{[1 \mid 5] \rightarrow 0} s_{15} A_{[1 \mid 3][1 \mid 5]} & =-\frac{\langle 1 \mid 5\rangle^{2}\langle 3 \mid 4\rangle[4 \mid 5][2 \mid 4]^{5}}{[2 \mid 3][3 \mid 4][3 \mid 5][2 \mid 5]^{2}} \\
& =\frac{\langle 1 \mid 5\rangle^{2}\langle 3 \mid 2\rangle[2 \mid 5][2 \mid 4]^{5}}{[2 \mid 3][3 \mid 4][3 \mid 5][2 \mid 5]^{2}} \\
& =-\frac{\langle 1 \mid 5\rangle^{2}\langle 2 \mid 3\rangle[2 \mid 4]^{5}}{[2 \mid 3][2 \mid 5][3 \mid 4][3 \mid 5]} .
\end{aligned}
$$

Then we have

$$
\begin{aligned}
A_{[1 \mid 3][1 \mid 5]}^{\prime}= & \frac{\langle 1 \mid 3\rangle\langle 2 \mid 5\rangle[2 \mid 4]^{5}}{[1 \mid 3][2 \mid 3][2 \mid 5][3 \mid 5][4 \mid 5]} \\
& +\frac{\langle 1 \mid 5\rangle\langle 2 \mid 3\rangle[2 \mid 4]^{5}}{[1 \mid 5][2 \mid 3][2 \mid 5][3 \mid 4][3 \mid 5]},
\end{aligned}
$$

which will be our starting expression for later calculations.

Since $A_{[1 \mid 3][1 \mid 5]}$ does not contain the pole [1|2], we must add a term to give the correct factorization limit for [1|2] $\rightarrow$ 0 . Thus we consider [1|2] $\rightarrow 0$, and we get

$$
\begin{aligned}
\lim _{[1 \mid 2] \rightarrow 0} A_{L} A_{R} & =-\frac{\langle 1 \mid 2\rangle^{2}\langle 3 \mid 5\rangle[1 \mid 4]^{2}[2 \mid 4]^{4}}{[1 \mid 3][1 \mid 5][3 \mid 4][3 \mid 5][4 \mid 5]} \\
& =-\frac{\langle 1 \mid 2\rangle^{2}\langle 3 \mid 5\rangle[2 \mid 4]^{6}}{[2 \mid 3][2 \mid 5][3 \mid 4][3 \mid 5][4 \mid 5]}, \\
A_{[1 \mid 2]} & =-\frac{\lim _{[1 \mid 2] \rightarrow 0} A_{L} A_{R}}{s_{12}} \\
& =-\frac{\langle 1 \mid 2\rangle\langle 3 \mid 5\rangle[2 \mid 4]^{6}}{[1 \mid 2][2 \mid 3][2 \mid 5][3 \mid 4][3 \mid 5][4 \mid 5]} .
\end{aligned}
$$

The purpose of the last step in the first line is to deform properly under the limit, so that the poles [1|3] and [1|5] in the denominator can be removed to keep the factorization limits for [1|3] $\rightarrow 0$ and [1|5] $\rightarrow 0$ when $A_{[1 \mid 3][1 \mid 5]}^{\prime}$ is added. Now we arrive at

$$
\begin{aligned}
A_{[1 \mid 2][1 \mid 3][1 \mid 5]}= & \frac{\langle 1 \mid 3\rangle\langle 2 \mid 5\rangle[2 \mid 4]^{5}}{[1 \mid 3][2 \mid 3][2 \mid 5][3 \mid 5][4 \mid 5]} \\
& +\frac{\langle 1 \mid 5\rangle\langle 2 \mid 3\rangle[2 \mid 4]^{5}}{[1 \mid 5][2 \mid 3][2 \mid 5][3 \mid 4][3 \mid 5]} \\
& -\frac{\langle 1 \mid 2\rangle\langle 3 \mid 5\rangle[2 \mid 4]^{6}}{[1 \mid 2][2 \mid 3][2 \mid 5][3 \mid 4][3 \mid 5][4 \mid 5]},
\end{aligned}
$$

which has the correct factorization limits for the poles [1|3], [1|5], [1|2]. One can observe that it is invariant when exchanging 3 and 5. It can be checked that it gives the correct factorization limits for remain channels, therefore

$A_{5}\left(1_{\gamma}^{-1}, 2_{\gamma}^{+1}, 3_{g}^{-2}, 4_{g}^{+2}, 5_{g}^{-2}\right)=A_{[1 \mid 2][1 \mid 3][1 \mid 5] .}$

The calculation above starts from a deformation which yields a non-zero boundary contribution. In order to verify (86), we can calculate the amplitude by the BCFW approach under a correct deformation, and compare the result with (86). Let us choose the deformation as

$\tilde{\lambda}_{3} \rightarrow \tilde{\lambda}_{3}+z \tilde{\lambda}_{5}, \quad \lambda_{5} \rightarrow \lambda_{5}-z \lambda_{3}$.

Then the BCFW approach gives

$$
\begin{aligned}
A_{5}\left(1_{\gamma}^{-1}, 2_{\gamma}^{+1}, 3_{g}^{-2}, 4_{g}^{+2}, 5_{g}^{-2}\right) \\
=\frac{[1 \mid 4][2 \mid 4]^{5}}{[1 \mid 2][3 \mid 5]^{2}}\left(\frac{\langle 1 \mid 3\rangle\langle 2 \mid 4\rangle}{[1 \mid 3][2 \mid 5][4 \mid 5]}\right. \\
\left.\quad+\frac{\langle 3 \mid 2\rangle\langle 1 \mid 4\rangle}{[1 \mid 5][2 \mid 3][4 \mid 5]}+\frac{\langle 1 \mid 2\rangle\langle 3 \mid 4\rangle}{[1 \mid 5][2 \mid 5][3 \mid 4]}\right) .
\end{aligned}
$$

One can verify that it is equal to (86) although their expressions look totally different.

5.4 The five-point amplitude $A_{5}\left(1_{\gamma}^{-1}, 2_{\gamma}^{+1}, 3_{\gamma}^{-1}, 4_{\gamma}^{+1}, 5_{g}^{-2}\right)$

The next example is the five-point amplitude $A_{5}\left(1_{\gamma}^{-1}, 2_{\gamma}^{+1}\right.$, $3_{\gamma}^{-1}, 4_{\gamma}^{+1}, 5_{g}^{-2}$ ). As in the previous case, a brief analysis of sub-amplitudes shows that all poles are of the form $[\bullet \mid \bullet]$. We again start from the result given by the BCFW approach under 
a wrong deformation which contains the non-zero boundary term. Such a deformation can be chosen as

$\tilde{\lambda}_{1} \rightarrow \tilde{\lambda}_{1}-z \tilde{\lambda}_{2}, \quad \lambda_{2} \rightarrow \lambda_{2}+z \lambda_{1}$,

then the BCFW approach gives

$$
\begin{aligned}
A_{[1 \mid 4][1 \mid 5]}= & -\frac{\langle 1 \mid 4\rangle\langle 3 \mid 5\rangle[3 \mid 4][2 \mid 4]^{4}}{[1 \mid 4][2 \mid 3][2 \mid 5][3 \mid 5][4 \mid 5]} \\
& +\frac{\langle 1 \mid 5\rangle\langle 3 \mid 4\rangle[3 \mid 5][2 \mid 4]^{5}}{[1 \mid 5][2 \mid 3][3 \mid 4][4 \mid 5][2 \mid 5]^{2}},
\end{aligned}
$$

which produces the correct factorization limits for the poles [1|4] and [1|5]. Again the presence of the double pole [2|5] suggests us to deform it. To do so, we can use $\lim _{[1 \mid 5] \rightarrow 0}\langle 3 \mid 4\rangle[3 \mid 5]=-\langle 2 \mid 4\rangle[2 \mid 5]$ to rewrite the second term as

$$
\frac{\langle 1 \mid 5\rangle\langle 3 \mid 4\rangle[3 \mid 5][2 \mid 4]^{5}}{[1 \mid 5][2 \mid 3][3 \mid 4][4 \mid 5][2 \mid 5]^{2}} \rightarrow-\frac{\langle 1 \mid 5\rangle\langle 2 \mid 4\rangle[2 \mid 4]^{5}}{[1 \mid 5][2 \mid 3][3 \mid 4][4 \mid 5][2 \mid 5]} .
$$

Thus we get our starting expression,

$A_{[1 \mid 4][1 \mid 5]}^{\prime}=A_{[1 \mid 4]}+A_{[1 \mid 5]}$,

with

$$
\begin{aligned}
& A_{[1 \mid 4]}=-\frac{\langle 1 \mid 4\rangle\langle 3 \mid 5\rangle[3 \mid 4][2 \mid 4]^{4}}{[1 \mid 4][2 \mid 3][2 \mid 5][3 \mid 5][4 \mid 5]}, \\
& A_{[1 \mid 5]}=-\frac{\langle 1 \mid 5\rangle\langle 2 \mid 4\rangle[2 \mid 4]^{5}}{[1 \mid 5][2 \mid 3][3 \mid 4][4 \mid 5][2 \mid 5]} .
\end{aligned}
$$

Then we try to include a term to produce the correct factorization limit for the pole [1|2], which is not contained in $A_{[1 \mid 4][1 \mid 5]}^{\prime}$. The symmetry of exchanging labels implies $A_{[1 \mid 2]}$ can be obtained by exchanging 2 and 4 in $A_{[1 \mid 4]}$, thus we arrive at

$$
\begin{aligned}
A_{[1 \mid 2][1 \mid 4][1 \mid 5]}= & A_{[1 \mid 2]}+A_{[1 \mid 4][1 \mid 5]}^{\prime} \\
= & -\frac{\langle 1 \mid 2\rangle\langle 3 \mid 5\rangle[2 \mid 3][2 \mid 4]^{4}}{[1 \mid 2][3 \mid 4][2 \mid 5][3 \mid 5][4 \mid 5]} \\
& -\frac{\langle 1 \mid 4\rangle\langle 3 \mid 5\rangle[3 \mid 4][2 \mid 4]^{4}}{[1 \mid 4][2 \mid 3][2 \mid 5][3 \mid 5][4 \mid 5]} \\
& -\frac{\langle 1 \mid 5\rangle\langle 2 \mid 4\rangle[2 \mid 4]^{5}}{[1 \mid 5][2 \mid 3][3 \mid 4][4 \mid 5][2 \mid 5]} .
\end{aligned}
$$

Now we consider the factorization limit for the pole [3|4]. $A_{[3 \mid 4]}$ can be obtained by exchanging 1 and 3 in $A_{[1 \mid 4]}$ :

$$
A_{[3 \mid 4]}=\frac{\langle 1 \mid 5\rangle\langle 3 \mid 4\rangle[1 \mid 4][2 \mid 4]^{4}}{[3 \mid 4][1 \mid 2][2 \mid 5][1 \mid 5][4 \mid 5]},
$$

thus using (4) one can construct

$$
\begin{aligned}
A_{[3 \mid 4]}^{\prime}= & A_{[1 \mid 2][1 \mid 4][1 \mid 5]} \\
& +\left(A_{[3 \mid 4]}-\frac{\lim _{[3 \mid 4] \rightarrow 0} s_{34} A_{[1 \mid 2][1 \mid 4][1 \mid 5]}}{s_{34}}\right) \\
= & A_{[3 \mid 4]}+A_{[1 \mid 4]} .
\end{aligned}
$$

$A_{[3 \mid 4]}^{\prime}$ gives the correct factorization limits for [3|4] $\rightarrow 0$ and $[1 \mid 4] \rightarrow 0$, but no longer has the correct factorization limits for [1|2] $\rightarrow 0$ and [1|5] $\rightarrow 0$. This is a phenomenon one will encounter if the representative expression is not properly chosen as mentioned in Sect. 2. To avoid this, one needs to find proper representative expressions for $A_{[3 \mid 4]}$ in (95) and $A_{[1 \mid 4]}$ in (93), such that at each iterative step, the correct factorization limit is satisfied not only for the new pole, but also for other poles in previous steps.

Now we try to deform $A_{[3 \mid 4]}$ in (95). Noting that $A_{[3 \mid 4]}$ contains both poles [1|2] and [3|4], we try to transform it so that it gives the correct factorization limit for the pole [1|2] automatically. Using

$$
\begin{aligned}
\lim _{[3 \mid 4] \rightarrow 0} s_{34} A_{[3 \mid 4]} & =-\frac{\langle 1 \mid 5\rangle\langle 3 \mid 4\rangle^{2}[1 \mid 4][2 \mid 4]^{4}}{[1 \mid 2][2 \mid 5][1 \mid 5][4 \mid 5]} \\
& =\frac{\langle 2 \mid 5\rangle\langle 3 \mid 4\rangle^{2}[3 \mid 5][2 \mid 4]^{5}}{[1 \mid 2][2 \mid 5][1 \mid 5][4 \mid 5][3 \mid 5]} \\
& =-\frac{\langle 1 \mid 2\rangle\langle 3 \mid 4\rangle^{2}[1 \mid 3][2 \mid 4]^{5}}{[1 \mid 2][1 \mid 5][2 \mid 5][3 \mid 5][4 \mid 5]}
\end{aligned}
$$

and

$$
\begin{aligned}
\lim _{[1 \mid 2] \rightarrow 0} s_{12} A_{[1 \mid 2]} & =\frac{\langle 1 \mid 2\rangle^{2}\langle 3 \mid 5\rangle[2 \mid 3][2 \mid 4]^{4}}{[3 \mid 4][2 \mid 5][3 \mid 5][4 \mid 5]} \\
& =-\frac{\langle 1 \mid 2\rangle^{2}\langle 4 \mid 5\rangle[1 \mid 5][2 \mid 4]^{5}}{[3 \mid 4][2 \mid 5][3 \mid 5][4 \mid 5][1 \mid 5]} \\
& =-\frac{\langle 1 \mid 2\rangle^{2}\langle 3 \mid 4\rangle[1 \mid 3][2 \mid 4]^{5}}{[1 \mid 5][2 \mid 5][3 \mid 4][3 \mid 5][4 \mid 5]},
\end{aligned}
$$

we obtain

$$
\begin{aligned}
A_{[1 \mid 2][3 \mid 4]} & =A_{[1 \mid 2]}=A_{[3 \mid 4]} \\
& =\frac{\langle 1 \mid 2\rangle\langle 3 \mid 4\rangle[1 \mid 3][2 \mid 4]^{5}}{[1 \mid 2][1 \mid 5][2 \mid 5][3 \mid 4][3 \mid 5][4 \mid 5]},
\end{aligned}
$$

where $A_{[1 \mid 2]}$ in (94) has been deformed as well. Using the deformed $A_{[3 \mid 4]}$ in (99) from (94) to (96), we find that the new $A_{[3 \mid 4]}^{\prime}$ gives the correct factorization limits for the poles [3|4], [1|4], and [1|2], but not for the pole [1|5]. To fix this problem, we need to deform $A_{[1 \mid 4]}$ or $A_{[1 \mid 5]}$ in (93).

Now noticing the symmetry $1 \leftrightarrow 3$ or $2 \leftrightarrow 4$, we can construct $A_{[1 \mid 4][2 \mid 3]}$ from $A_{[1 \mid 2][3 \mid 4]}$ in (99) by exchanging 2 and 4 as

$$
\begin{aligned}
A_{[1 \mid 4][2 \mid 3]} & =A_{[1 \mid 4]}=A_{[2 \mid 3]} \\
& =-\frac{\langle 1 \mid 4\rangle\langle 2 \mid 3\rangle[1 \mid 3][2 \mid 4]^{5}}{[1 \mid 4][1 \mid 5][2 \mid 3][2 \mid 5][3 \mid 5][4 \mid 5]} .
\end{aligned}
$$

Putting the new expressions in (99) and (100) into (96), we arrive at

$A_{[1 \mid 2][1 \mid 4][2 \mid 3][3 \mid 4]}=A_{[1 \mid 2][3 \mid 4]}+A_{[1 \mid 4][2 \mid 3]}$,

which gives the correct factorization limits for [1|2] $\rightarrow 0$, $[1 \mid 4] \rightarrow 0,[2 \mid 3] \rightarrow 0,[3 \mid 4] \rightarrow 0$ as well as [1|5] $\rightarrow 0$. To check it, notice that 
$\lim _{[1 \mid 5] \rightarrow 0} s_{15} A_{[1 \mid 2][1 \mid 4][2 \mid 3][3 \mid 4]}$

$$
\begin{aligned}
= & \frac{\langle 1 \mid 5\rangle\langle 1 \mid 4\rangle\langle 2 \mid 3\rangle[1 \mid 3][2 \mid 4]^{5}}{[1 \mid 4][2 \mid 3][2 \mid 5][3 \mid 5][4 \mid 5]} \\
& -\frac{\langle 1 \mid 5\rangle\langle 1 \mid 2\rangle\langle 3 \mid 4\rangle[1 \mid 3][2 \mid 4]^{5}}{[1 \mid 2][2 \mid 5][3 \mid 4][3 \mid 5][4 \mid 5]} \\
= & \frac{\langle 1 \mid 5\rangle\langle 2 \mid 4\rangle[2 \mid 4]^{5}}{[2 \mid 5][3 \mid 5][4 \mid 5]}\left(\frac{\langle 1 \mid 2\rangle}{[3 \mid 4]}-\frac{\langle 1 \mid 4\rangle}{[2 \mid 3]}\right) \\
= & \frac{\langle 1 \mid 5\rangle\langle 2 \mid 4\rangle[2 \mid 4]^{5}\langle 1|2+4| 3]}{[2 \mid 3][2 \mid 5][3 \mid 4][3 \mid 5][4 \mid 5]} \\
= & \frac{\langle 1 \mid 5\rangle\langle 2 \mid 4\rangle[2 \mid 4]^{5}\langle 1 \mid 5\rangle[3 \mid 5]}{[2 \mid 3][2 \mid 5][3 \mid 4][3 \mid 5][4 \mid 5]}
\end{aligned}
$$

therefore

$$
\begin{aligned}
\lim _{[1 \mid 5] \rightarrow 0} s_{15} A_{[1 \mid 2][1 \mid 4][2 \mid 3][3 \mid 4]} & =s_{15} A_{[1 \mid 5]} \\
& =-\lim _{[1 \mid 5] \rightarrow 0} A_{L} A_{R} .
\end{aligned}
$$

One can verify that $A_{[1 \mid 2][1 \mid 4][2 \mid 3][3 \mid 4]}$ also gives the correct factorization limits for remaining channels. Thus we find the correct amplitude is

$A\left(1_{\gamma}^{-1}, 2_{\gamma}^{+1}, 3_{\gamma}^{-1}, 4_{\gamma}^{+1}, 5_{g}^{-2}\right)=A_{[1 \mid 2][1 \mid 4][2 \mid 3][3 \mid 4]}$.

Notice that the formula of $A_{[1 \mid 2][1 \mid 4][2 \mid 3][3 \mid 4]}$ is manifestly invariant when exchanging $1 \leftrightarrow 3$ and $2 \leftrightarrow 4$. The result (104) can be rewritten as by the conclusions in $[9,10]$. Although naive power counting of Feynman diagrams shows that the large $z$ behavior is $z^{0}$, using the result given in (105), one can observe that under the deformation of two photons of the same helicity, the boundary term will vanish.

\subsection{The six-point amplitude}

$$
A_{6}\left(1_{\gamma}^{-1}, 2_{\gamma}^{+1}, 3_{\gamma}^{-1}, 4_{\gamma}^{+1}, 5_{\gamma}^{-1}, 6_{\gamma}^{+1}\right)
$$

The final example is the six-point amplitude $A_{6}\left(1_{\gamma}^{-}, 2_{\gamma}^{+}, 3_{\gamma}^{-}\right.$, $\left.4_{\gamma}^{+}, 5_{\gamma}^{-}, 6_{\gamma}^{+}\right)$. This case is a little different from those in previous subsections. For previous cases, we know there exist some deformations that can make boundary terms vanish, so we do not need our approach in this paper to find them. However, for $A_{6}\left(1_{\gamma}^{-1}, 2_{\gamma}^{+1}, 3_{\gamma}^{-1}, 4_{\gamma}^{+1}, 5_{\gamma}^{-1}, 6_{\gamma}^{+1}\right)$ the situation is different. First, since there is no graviton, the results in [10] cannot be applied. Secondly, we know the boundary term is not zero when deforming two photons with opposite helicities. Thirdly, when two deformed photons have the same helicity, the large $z$ behavior is $z^{0}$ by naive power counting of Feynman diagrams. Thus, our approach becomes one of the useful approaches.

Let us consider the deformation

$\lambda_{1} \rightarrow \lambda_{1}-z \lambda_{3}, \quad \tilde{\lambda}_{3} \rightarrow \tilde{\lambda}_{3}+z \tilde{\lambda}_{1}$.

Under this deformation, the BCFW approach gives residues for the following poles: [1|2], [1|4], [1|6], $\langle 3 \mid 2\rangle,\langle 3 \mid 4\rangle,\langle 3 \mid 6\rangle$, $s_{124}, s_{125}, s_{126}, s_{145}, s_{146}, s_{156}$. A little algebra yields

$$
\begin{aligned}
A= & \frac{\langle 1 \mid 2\rangle\langle 4 \mid 6\rangle\langle 5|1+3| 2]^{5}(\langle 4 \mid 5\rangle[3 \mid 4][5 \mid 6]\langle 6|1+3| 2]-\langle 5 \mid 6\rangle[3 \mid 6][4 \mid 5]\langle 4|1+3| 2])}{[1 \mid 2]\langle 4 \mid 5\rangle\langle 5 \mid 6\rangle\langle 4|1+3| 2]\langle 6|1+3| 2]\langle 4|1+2| 3]\langle 5|1+2| 3]\langle 6|1+2| 3] s_{123}}+(2 \leftrightarrow 4)+(2 \leftrightarrow 6) \\
& +\frac{\langle 1 \mid 3\rangle^{4}[2 \mid 3][4 \mid 6]^{5}\langle 2|1+3| 5](\langle 1 \mid 4\rangle\langle 5 \mid 6\rangle[4 \mid 5]\langle 2|1+3| 6]-\langle 1 \mid 6\rangle\langle 4 \mid 5\rangle[5 \mid 6]\langle 2|1+3| 4])}{\langle 2 \mid 3\rangle[4 \mid 5][5 \mid 6]\langle 2|1+3| 4]\langle 2|1+3| 6]\langle 1|2+3| 4]\langle 1|2+3| 5]\langle 1|2+3| 6] s_{123}}+(2 \leftrightarrow 4)+(2 \leftrightarrow 6) \\
& +\frac{s_{24}\langle 5 \mid 6\rangle[3 \mid 5]\langle 1|2+4| 6]^{5}}{\langle 1 \mid 2\rangle\langle 1 \mid 4\rangle[3 \mid 6][5 \mid 6]\langle 1|2+4| 5]\langle 2|1+4| 3]\langle 4|1+2| 3] s_{124}}+(4 \leftrightarrow 6)+(2 \leftrightarrow 6) \\
& +\frac{s_{46}\langle 1 \mid 5\rangle^{5}[2 \mid 5][4 \mid 6]^{4}\langle 2|1+5| 3]}{\langle 1 \mid 2\rangle\langle 2 \mid 5\rangle[3 \mid 4][3 \mid 6]\langle 1|2+5| 4]\langle 1|2+5| 6]\langle 5|1+2| 3] s_{125}}+(2 \leftrightarrow 4)+(2 \leftrightarrow 6)
\end{aligned}
$$

$$
\begin{aligned}
& A\left(1_{\gamma}^{-1}, 2_{\gamma}^{+1}, 3_{\gamma}^{-1}, 4_{\gamma}^{+1}, 5_{g}^{-2}\right) \\
& =\frac{[1 \mid 3][2 \mid 4]^{5}(\langle 1 \mid 4\rangle[3 \mid 4][1|4+5| 3\rangle-[1 \mid 4]\langle 3 \mid 4\rangle\langle 1|4+5| 3])}{[1 \mid 2][1 \mid 4][1 \mid 5][2 \mid 3][2 \mid 5][3 \mid 4][3 \mid 5][4 \mid 5]} \\
& =\frac{[1 \mid 3][2 \mid 4]^{5}([1 \mid 4][3 \mid 5]\langle 1 \mid 5\rangle\langle 3 \mid 4\rangle-\langle 1 \mid 4\rangle\langle 3 \mid 5\rangle[1 \mid 5][3 \mid 4])}{[1 \mid 2][1 \mid 4][1 \mid 5][2 \mid 3][2 \mid 5][3 \mid 4][3 \mid 5][4 \mid 5]},
\end{aligned}
$$

which is the formula in [16].

As a side note, when two deformed particles are two photons of the same helicity, there is no general proof of its boundary behavior, since this situation cannot be covered
Four explicit terms above are residues for the poles [1|2], $\langle 2 \mid 3\rangle, s_{124}$, and $s_{125}$. Other terms can be obtained by exchanging labels. The result (107) will be our starting expression.

Now we need to include factorization limits for the poles not detected by the chosen deformation. It turns out that the result (107) is the correct amplitude that we are seeking, since it gives the correct factorization limits for all physical channels. The symmetry of exchanging labels makes the verification very easy. The amplitude has the $S_{3} \otimes S_{3}$ symmetry: $1 \leftrightarrow 3,3 \leftrightarrow 5,5 \leftrightarrow 1$, and $2 \leftrightarrow 4,4 \leftrightarrow 6$, 
$6 \leftrightarrow 2$. Its factorization limits will be restricted by this symmetry, for example, $\lim _{[2 \mid 3] \rightarrow 0} A_{L} A_{R}$ can be obtained by exchanging 1 and 3 in $\lim _{[1 \mid 2] \rightarrow 0} A_{L} A_{R}$. Equation (107) already has the correct factorization limits for the poles detected by the BCFW approach. If it is invariant when some labels are exchanged, more correct factorization limits will be satisfied. For instance, (107) provides the correct factorization limit for [1|2] $\rightarrow 0$. If we perform the exchange $1 \leftrightarrow 3$ for (107), the new expression gives the correct factorization limit for [2|3] $\rightarrow 0$, since $\lim _{[2 \mid 3] \rightarrow 0} A_{L} A_{R}$ can be obtained from $\lim _{[1 \mid 2] \rightarrow 0} A_{L} A_{R}$ by this exchange. Thus, if (107) has the symmetry $1 \leftrightarrow 3$, the correct factorization limit for [2|3] $\rightarrow 0$ is also satisfied. Consequently, if (107) has the $S_{3} \otimes S_{3}$ symmetry as the correct amplitude, all factorization limits will be given. In (107), the symmetry $\{2 \leftrightarrow 4,4 \leftrightarrow 6,6 \leftrightarrow 2\}$ is manifest. On the other hand, we have checked the symmetry $\{1 \leftrightarrow 3,3 \leftrightarrow 5,5 \leftrightarrow 1\}$ numerically. Furthermore, we have verified that all spurious poles are canceled, as we did in Sect. 4.2. Therefore (107) is the correct $A_{6}\left(1_{\gamma}^{-1}, 2_{\gamma}^{+1}, 3_{\gamma}^{-1}, 4_{\gamma}^{+1}, 5_{\gamma}^{-1}, 6_{\gamma}^{+1}\right)$.

\section{Example 4: Yukawa theory (elimination of spurious poles)}

In this section we will show how to treat the case that an expression has the correct factorization limits for all physical poles but contains spurious poles. We have not encountered such a phenomenon in previous examples. However, the situation will appear when considering the color-ordered amplitude of fermions coupling to scalars by the Yukawa coupling.

In this theory, three-point amplitudes are given by

$$
\begin{aligned}
& A_{3}\left(1^{+}, 2,3^{+}\right)=[1 \mid 3], \quad A_{3}\left(1^{-}, 2,3^{-}\right)=\langle 1 \mid 3\rangle, \\
& A_{3}\left(1^{+}, 2,3^{-}\right)=A_{3}\left(1^{-}, 2,3^{+}\right)=0,
\end{aligned}
$$

where the coupling constant has been neglected. Let us consider the simplest case, the four-point amplitude $A_{4}\left(1^{-}, 2\right.$, $3,4^{+}$). This amplitude corresponds to only one Feynman diagram and the result can be obtained by Feynman rules as ${ }^{7}$

$A_{4}\left(1^{-}, 2,3,4^{+}\right)=\frac{\left\langle 1\left|P_{34}\right| 4\right]}{s_{34}}=\frac{\langle 1 \mid 3\rangle}{\langle 4 \mid 3\rangle}=-\frac{[2 \mid 4]}{[2 \mid 1]}$.

Now we use our approach to reproduce this result. Physical poles come from $s_{12} \rightarrow 0$, and only the solution $\tilde{\lambda}_{2} \propto \tilde{\lambda}_{1}$ provides non-vanishing sub-amplitudes, thus there is only one physical pole [1|2]. Using the solution $I_{1}$ in (70), we get the factorization limit for this pole as

\footnotetext{
${ }^{7}$ Since the propagator $\frac{i P}{P^{2}}$ depends on the direction of $P$, we assume that the fermion line is from 4 to 1 and all external momenta are incoming.
}

$A_{[1 \mid 2]}=-\frac{\lim _{[1 \mid 2] \rightarrow 0} A_{L} A_{R}}{s_{12}}=\frac{s_{14}}{\langle 2 \mid 4\rangle[1 \mid 2]}$.

The expression $A_{[1 \mid 2]}$ which has the correct factorization limit for the physical pole but also contains a spurious pole $\langle 2 \mid 4\rangle$. To eliminate it, let us use the approach discussed in Sect. 2, i.e., choose a correct expression for $\frac{\lim _{\{2|4\rangle \rightarrow 0}\langle 2 \mid 4\rangle A_{\langle 1 \mid 2\rangle}}{\langle 2 \mid 4\rangle}$ so that it does not contain any physical pole. This procedure can be performed:

$$
\lim _{\langle 2 \mid 4\rangle \rightarrow 0}\langle 2 \mid 4\rangle A_{\langle 1 \mid 2\rangle}=\lim _{\langle 2 \mid 4\rangle \rightarrow 0} \frac{s_{14}}{[1 \mid 2]}=\frac{-s_{12}}{[1 \mid 2]}=\langle 1 \mid 2\rangle .
$$

Then we can construct

$$
\begin{aligned}
A_{[1 \mid 2]}^{\prime} & =A_{[1 \mid 2]}-\frac{\lim _{\langle 2 \mid 4\rangle \rightarrow 0}\langle 2 \mid 4\rangle A_{\langle 1 \mid 2\rangle}}{\langle 2 \mid 4\rangle} \\
& =\frac{-s_{13}}{\langle 2 \mid 4\rangle[1 \mid 2]}=\frac{[2 \mid 4]}{[1 \mid 2]},
\end{aligned}
$$

which is the correct answer.

However, the above approach for removing spurious poles will be difficult to perform if the amplitude contains many physical poles. We have not found a more efficient approach.

\section{Conclusion}

In this paper, we have proposed an approach to calculate tree amplitudes without polynomial terms through their factorization limits. We seek a quantity that has the correct factorization limits for all physical poles and does not contain other poles iteratively. Starting from an initial function which has the correct factorization limits for some poles, we adjust our expression to include factorization limits for new channels at each iterative step, while keeping the correct factorization limits of the previous poles. Proceeding thus, a proper choice of the expression in the equivalent category under corresponding limits is required. We have shown how to make such a choice in various examples. Because at each step at least one new pole is included into the set of channels having the correct factorization limits, this algorithm will stop at finite steps. After obtaining an expression which has the correct factorization limits for all physical poles, we need to eliminate possible spurious poles. Then we get the desired result. This approach can be applied to all circumstances no matter whether the boundary contribution vanishes. However, this approach cannot determine the polynomial terms since it can only detect the pole part. If the amplitude admits polynomials which satisfy correct mass dimension and helicities, the full amplitude cannot be determined. Such polynomials can be included into the theory by allowing certain higher-dimensional coupling constants.

To demonstrate this, we have applied our approach to calculate amplitudes of $\phi^{4}$ theory, pure gauge theory, EinsteinMaxwell theory, and Yukawa theory. Correct results of these 
examples are obtained, although their calculations are somewhat complicated. In these examples, one can see that no information as regards boundary terms is required when using this approach.

In principle, one can split an amplitude into more than two sub-amplitudes by imposing on-shell conditions on more propagators. However, it will make the computation more complicated. For example, if we cut the amplitude into three sub-amplitudes, we need to consider factorization limits for all possible combinations of two channels. The number of such combinations grows extremely faster than the number of channels. The maximal cut is imposing on-shell conditions on all propagators, then all sub-amplitudes are lowest-point amplitudes. In such a case, all possible Feynman diagrams need to be considered one by one.

It is interesting to consider whether this approach can be generalized to tree amplitudes of string theory. The most difficult issue is, in string theory, that the number of inner states is infinite. We have not found a way to tackle this difficulty.

In this paper, all examples are within consistent quantum field theories. Another important direction in the future is to apply this approach to any theory which is known to be inconsistent. An example is massless spin-3 fields: here the three-point amplitudes are known, but on general grounds no higher-point tree-level amplitudes should be 'constructable', thus our approach encounters the difficulty. Also, coupling gauge or gravity fields to higher spin $(>2)$ massive or massless matter would be interesting.

Acknowledgments The authors would like to thank Prof. Bo Feng for his guidance throughout the project. This work is supported by Chinese NSF funding under contracts No.11031005, No.11135006 and No.11125523.

Open Access This article is distributed under the terms of the Creative Commons Attribution 4.0 International License (http://creativecomm ons.org/licenses/by/4.0/), which permits unrestricted use, distribution, and reproduction in any medium, provided you give appropriate credit to the original author(s) and the source, provide a link to the Creative Commons license, and indicate if changes were made. Funded by SCOAP ${ }^{3}$.

\section{Appendix A: Absence of polynomials}

Here we give a brief proof of the fact that the amplitudes calculated in this paper do not contain any polynomial term. The argument is simple: One cannot construct a polynomial that has the correct mass dimension of the amplitude and the correct helicities of all external particles.

The mass dimension of a bare amplitude is $D=4-$ $n-\sum D_{c}$. Here $D_{c}$ are mass dimensions of coupling constants. When we mention the 'bare amplitude', we mean that all coupling constants are stripped off. For amplitudes of $\phi^{4}$ theory, pure gauge theory, and Yukawa theory, since the coupling constants are dimensionless, we have $D<0$ if the amplitude contains at least five external particles, and $D=0$ if the amplitude contains four external particles. Thus, the only possible polynomial is a constant which corresponds to four-point amplitudes. However, a constant cannot provide the correct helicities for external particles, unless all external states are scalars. Consequently, for such amplitudes, the only allowed polynomial is just the lowest-point amplitude of $\phi^{4}$ theory.

For amplitudes of Einstein-Maxwell theory, the coupling constant has mass dimension -1 , thus for all amplitudes of this theory we have $D=-2$ for an arbitrary $n$. Then the possible polynomial can take the form $\langle\bullet \mid \bullet\rangle\langle\bullet \mid \bullet\rangle,[\bullet \mid \bullet][\bullet \mid \bullet]$, and $\langle\bullet \mid \bullet\rangle[\bullet \mid \bullet]$. None of these can provide correct helicities for all external states since one spinor can only carry helicity $\pm \frac{1}{2}$, and the amplitudes we have calculated contain at least four external particles (recall that a photon has helicity \pm 1 , one graviton has helicity \pm 2 , and for spinorial products, $\langle i \mid i\rangle=0, \quad[i \mid i]=0)$.

Hence, the amplitudes mentioned in this paper do not contain any polynomial term.

Appendix B: Alternative calculation of $A_{6}\left(1^{-}, 2^{-}, 3^{-}\right.$, $\left.4^{+}, 5^{+}, 6^{+}\right)$

In this section, we present the calculation of $A_{6}\left(1^{-}, 2^{-}, 3^{-}\right.$, $4^{+}, 5^{+}, 6^{+}$) that starts by considering the factorization limit for the three-particle channel $s_{234} \rightarrow 0$. Unlike the twoparticle channel where the on-shell limit is split into the holomorphic and anti-holomorphic parts, the on-shell limit of $s_{234} \rightarrow 0$ could not be split, therefore $s_{234}$ will appear in the denominator of the amplitude as a whole. Thus we do not solve the constraint on the kinematic variables, as in the calculation in the $\phi^{4}$ case. The factorization limit for $s_{234} \rightarrow 0$ is given by

$$
\begin{gathered}
\lim _{s_{234} \rightarrow 0} A_{4}\left(2^{-}, 3^{-}, 4^{+},-P_{234}^{+}\right) A_{4}\left(P_{234}^{-}, 5^{+}, 6^{+}, 1^{-}\right) \\
=\frac{\langle 2 \mid 3\rangle^{3}}{\langle 3 \mid 4\rangle\left\langle 4 \mid P_{234}\right\rangle\left\langle P_{234} \mid 2\right\rangle} \frac{\left\langle 1 \mid P_{234}\right\rangle^{3}}{\left\langle P_{234} \mid 5\right\rangle\langle 5 \mid 6\rangle\langle 6 \mid 1\rangle} .
\end{gathered}
$$

To express $\lambda_{P_{234}}$, we can use $\left\langle a \mid P_{234}\right\rangle=\frac{\left\langle a\left|P_{234}\right| b\right]}{\left[P_{234} \mid b\right]}$. The calculation is as follows:

$$
\begin{aligned}
\lim _{s_{234} \rightarrow 0} A_{4}\left(2^{-}, 3^{-}, 4^{+},-P_{234}^{+}\right) A_{4}\left(P_{234}^{-}, 5^{+}, 6^{+}, 1^{-}\right) \\
=\frac{\langle 2 \mid 3\rangle^{3}}{\langle 3 \mid 4\rangle\left\langle 4 \mid P_{234}\right\rangle\left\langle P_{234} \mid 2\right\rangle} \frac{\left\langle 1 \mid P_{234}\right\rangle^{3}}{\left\langle P_{234} \mid 5\right\rangle\langle 5 \mid 6\rangle\langle 6 \mid 1\rangle} \\
\quad \times \frac{\left[P_{234} \mid 2\right]^{2}\left[P_{234} \mid 4\right]}{\left[P_{234} \mid 2\right]^{2}\left[P_{234} \mid 4\right]}
\end{aligned}
$$




$$
\begin{aligned}
& =\frac{\left\langle 1\left|P_{234}\right| 4\right]\left\langle 1\left|P_{234} 2\right| 3\right\rangle^{2}}{\langle 5 \mid 6\rangle\langle 6 \mid 1\rangle\langle 3 \mid 4\rangle^{2}[2 \mid 3][3 \mid 4]\left\langle 5\left|P_{234}\right| 2\right]} \\
& =\frac{\langle 1|2+3| 4]\left\langle 1\left|P_{234} 4\right| 3\right\rangle^{2}}{\langle 5 \mid 6\rangle\langle 6 \mid 1\rangle\langle 3 \mid 4\rangle^{2}[2 \mid 3][3 \mid 4]\langle 5|3+4| 2]} \\
& =\frac{\langle 1|2+3| 4]^{3}}{\langle 5 \mid 6\rangle\langle 6 \mid 1\rangle[2 \mid 3][3 \mid 4]\langle 5|3+4| 2]} .
\end{aligned}
$$

Going from the second line to the third line, we have used $\left\langle 1\left|P_{234} 2\right| 3\right\rangle=\left\langle 1\left|P_{234}(2+3)\right| 3\right\rangle=-\left\langle 1\left|P_{234} 4\right| 3\right\rangle$. This step is necessary if we try to include correct factorization limits for the poles $\langle 5 \mid 6\rangle$ and [2|3]. Thus we obtain

$$
A_{\langle 5 \mid 6\rangle[2 \mid 3] s_{234}}=-\frac{\langle 1|2+3| 4]^{3}}{\langle 5 \mid 6\rangle\langle 6 \mid 1\rangle[2 \mid 3][3 \mid 4]\langle 5|3+4| 2] s_{234}} .
$$

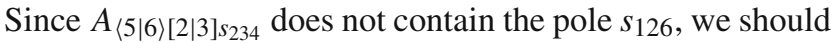
compute the factorization limit for this pole and add it to

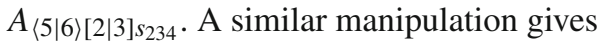

$$
A_{\langle 4 \mid 5\rangle[1 \mid 2] s_{126}}=-\frac{\langle 3|4+5| 6]^{3}}{\langle 3 \mid 4\rangle\langle 4 \mid 5\rangle[1 \mid 2][6 \mid 1]\langle 5|3+4| 2] s_{126}} .
$$

Summing (B3) and (B4), we arrive at the correct result.

\section{References}

1. E. Witten, Commun. Math. Phys. 252, 189 (2004). arXiv:hep-th/0312171

2. R. Britto, F. Cachazo, B. Feng, Nucl. Phys. B 715, 499 (2005). arXiv:hep-th/0412308
3. R. Britto, F. Cachazo, B. Feng, E. Witten, Phys. Rev. Lett. 94, 181602 (2005). arXiv:hep-th/0501052

4. Z. Bern, L.J. Dixon, D.A. Kosower, Ann. Phys. 322, 1587 (2007). arXiv:0704.2798 [hep-ph]

5. B. Feng, M. Luo, Front. Phys. 7, 533 (2012). arXiv:1111.5759 [hep-th]

6. H. Elvang, Y.T. Huang. arXiv:1308.1697 [hep-th]

7. K. Risager, JHEP 0512, 003 (2005). arXiv:hep-th/0508206

8. P. Benincasa, C. Boucher-Veronneau, F. Cachazo, JHEP 0711, 057 (2007). arXiv:hep-th/0702032 [hep-th]

9. N. Arkani-Hamed, J. Kaplan, JHEP 0804, 076 (2008). arXiv:0801.2385 [hep-th]

10. C. Cheung, JHEP 1003, 098 (2010). arXiv:0808.0504 [hep-th]

11. P. Benincasa, F. Cachazo. arXiv:0705.4305 [hep-th]

12. R.H. Boels, JHEP 1005, 046 (2010). arXiv:1003.2989 [hep-th]

13. B. Feng, J. Wang, Y. Wang, Z. Zhang, JHEP 1001, 019 (2010). arXiv:0911.0301 [hep-th]

14. B. Feng, C.Y. Liu, JHEP 1007, 093 (2010). arXiv:1004.1282 [hepth]

15. B. Feng, Z. Zhang, JHEP 1112, 057 (2011). arXiv:1109.1887 [hepth]

16. P. Benincasa, E. Conde, JHEP 1111, 074 (2011). arXiv: 1106.0166 [hep-th]

17. P. Benincasa, E. Conde, Phys. Rev. D 86, 025007 (2012). arXiv:1108.3078 [hep-th]

18. B. Feng, Y. Jia, H. Luo, M. Luo. arXiv:1111.1547 [hep-th] 